\title{
WEAKLY COUPLED MEAN-FIELD GAME SYSTEMS
}

\author{
DIOGO A. GOMES AND STEFANIA PATRIZI
}

\begin{abstract}
Here, we prove the existence of solutions to first-order mean-field games (MFGs) arising in optimal switching. First, we use the penalization method to construct approximate solutions. Then, we prove uniform estimates for the penalized problem. Finally, by a limiting procedure, we obtain solutions to the MFG problem.
\end{abstract}

\section{INTRODUCTION}

The mean-field game (MFG) framework [34, 35, 36, 37, 38, 39] is a class of methods used to study large populations of rational, non-cooperative agents. MFGs have been the focus of intense research, see, for example, the surveys [28, 31]. Here, we investigate MFGs that arise in optimal switching. These games are given by a weakly coupled system of Hamilton-Jacobi equations of the obstacle type and a corresponding system of transport equations.

To simplify the presentation, we use periodic boundary conditions. Thus, the spatial domain is the $N$-dimensional flat torus, $\mathbb{T}^{N}$. Our MFG is determined by a value function, $u: \mathbb{T}^{N} \rightarrow \mathbb{R}^{d}$, a probability density, $\theta: \mathbb{T}^{N} \rightarrow\left(\mathbb{R}^{+}\right)^{d}$, and a switching current, $\nu$, that together satisfy the following system of variational inequalities:

$$
\max \left(H^{i}\left(D u^{i}, x\right)+u^{i}-g\left(\theta^{i}\right), \max _{j}\left(u^{i}-u^{j}-\psi^{i j}\right)\right)=0
$$

coupled with the system

$$
-\operatorname{div}\left(D_{p} H^{i}\left(D u^{i}, x\right) \theta^{i}\right)+\theta^{i}+\sum_{j \neq i}\left(\nu^{i j}-\nu^{j i}\right)=1 .
$$

Moreover, for $1 \leq i, j \leq d, \nu^{i j}$ is a non-negative measure on $\mathbb{T}^{N}$ supported in the set $u^{i}-u^{j}-\psi^{i j}=0$.

This system models a stationary population of agents. Each agent moves in $\mathbb{T}^{N}$ and can switch between different modes that are given by the index $i$. Their actions seek to minimize a certain cost. Agents can change their state by continuously modifying their spatial position, $x$, and by switching between different modes, $i$ to $j$, at a cost $\psi^{j i}$. The function $u^{i}(x)$ is the value function for an agent whose spatial location is $x$ and whose mode is $i$. The function $\theta^{i}(x)$ is the density of the agents on $\mathbb{T}^{N} \times\{1, \ldots, d\}$. Thus, we require that $\theta^{i}(x) \geq 0$. We note that $\theta^{i}$ is not a

Date: May 23, 2016.

2010 Mathematics Subject Classification. 35J47, 35A01.

Key words and phrases. Mean Field Games; Weakly coupled systems; Optimal Switching.

D. Gomes was partially supported by KAUST baseline and start-up funds. S. Patrizi was partially supported by NSF grant DMS-1262411 "Regularity and stability results in variational problems". 
probability measure on $\mathbb{T}^{N} \times\{1, \ldots, d\}$ because the source term in the right-hand side of (1.2) is not normalized.

In Section 2, we discuss detailed assumptions on the Hamiltonians $H^{i}$, on the nonlinearity $g$, and on the switching costs $\psi^{i j}$. A concrete example that satisfies those is

$$
H^{i}(x, p)=\frac{|p|^{2}}{2}+V^{i}(x), \quad g(\theta)=\ln \theta, \quad \text { and } \quad \psi^{i j}(x)=\eta,
$$

with $V^{i}: \mathbb{T}^{N} \rightarrow \mathbb{R}$ being a $C^{\infty}$ function and $\eta$ being a positive real number. Another case of interest is the polynomial nonlinearity, $g(m)=m^{\alpha}$ for $\alpha>0$.

Standard MFGs involve two equations, a Hamilton-Jacobi equation and a transport or Fokker-Planck equation. This latter equation is the adjoint of the linearization of the former. Because the non-linear operator in (1.1) is non-differentiable, (1.2) is obtained by a limiting procedure. In the context of MFGs, this method was first used in [22]. Here, we consider the following penalized problem.

$$
\begin{gathered}
H^{i}\left(D u^{i}, x\right)+u^{i}+\sum_{j \neq i} \beta_{\epsilon}\left(u^{i}-u^{j}-\psi^{i j}\right)=g\left(\theta^{i}\right) \\
-\operatorname{div}\left(D_{p} H^{i}\left(D u^{i}, x\right) \theta^{i}\right)+\theta^{i}+\sum_{j \neq i} \beta_{\epsilon}^{\prime}\left(u^{i}-u^{j}-\psi^{i j}\right) \theta^{i}-\beta_{\epsilon}^{\prime}\left(u^{j}-u^{i}-\psi^{j i}\right) \theta^{j}=1,
\end{gathered}
$$

where the penalty function, $\beta_{\epsilon}$, is an increasing $C^{\infty}$ function and $\epsilon>0$. We assume that, as $\epsilon \rightarrow 0, \beta_{\epsilon}(s) \rightarrow \infty$ for $s>0$ and $\beta_{\epsilon}(s)=0$ for $s \leq 0$, see Assumption 8 . The study of optimal switching has a long history that predates viscosity solutions and, certainly, MFGs, see, for example [2, 6, 7, 17]. In those references, the use of a penalty to approximate a non-smooth Hamilton-Jacobi equation is a fundamental tool. The penalty in (1.4) is similar to the ones in the aforementioned references.

More recently, several authors have investigated weakly coupled Hamilton-Jacobi equations [44], the corresponding extension of the weak KAM and Aubry-Mather theories $[5,14,40]$, the asymptotic behavior of solutions $[4,3,42,41,45]$, and homogenization [43]. In these references, the state of the system has different modes, and a random process drives the switching between them. In contrast, here, the switches occur at deterministic times. Thus, our models are the MFG counterpart of the Hamilton-Jacobi systems considered in [18, 32]. MFGs with different populations $[12,13]$ are a limit case of (1.1)-(1.2). This can be seen by taking the limit $\psi^{i j} \rightarrow+\infty$; that is, the case where agents are not allowed to change their state.

The development of the existence and regularity theory for MFGs has seen substantial progress in recent years. Uniformly elliptic and parabolic MFGs are now well understood, and the existence of smooth and weak solutions has been established in a broad range of problems, see, respectively, [23, 24, 25, 30, 29, 27, 26] and $[10,46,47]$. However, the regularity theory for first-order MFGs is less developed and, in general, only weak solutions are known to exist $[8,9,11]$. Variational inequality methods are at the heart of a new class of techniques to establish the existence of weak solutions, both for first- and second-order problems [19] and for their numerical approximation [1]. Some MFGs that arise in applications, such as congestion [20,33] or obstacle-type problems [22], feature singularities. Thus, there is keen interest in developing methods for their analysis. To the best of our knowledge, this paper is the first to address MFGs arising in optimal switching. 
Moreover, our techniques contribute to better understanding of the regularity of first-order MFGs.

Our main result is the following theorem.

Theorem 1.1. Suppose that Assumptions 1-4 (see Section 2) hold and that either

- Assumption $5 \mathbf{L}$ or

- Assumptions $5 \mathbf{P}-\frac{2}{N}, 6$ and 7

hold. Then, there exists a solution, $(u, \theta)$, of (1.1)-(1.2), with $u \in\left(W^{2,2}\left(\mathbb{T}^{N}\right)\right)^{d} \cap$ $\left(C^{\gamma}\left(\mathbb{T}^{N}\right)\right)^{d}$ for some $\gamma \in(0,1)$ and $\theta \in\left(W^{1,2}\left(\mathbb{T}^{N}\right)\right)^{d}$.

As mentioned before, to prove the existence of solutions for (1.1)-(1.2), we first examine the existence of solutions for (1.4)-(1.5), prove $\epsilon$ independent bounds and, subsequently, consider the limit $\epsilon \rightarrow 0$. On the existence of solutions, our main result is the following theorem.

Theorem 1.2. Suppose that Assumptions 1-4 (see Section 2) and 8 hold, and either

- Assumption $5 \mathbf{L}$ or

- Assumptions $5 \mathrm{P}-\frac{2}{N}, 6$ and 7

hold. Then, there exists a unique solution, $(u, \theta) \in\left(C^{\infty}\left(\mathbb{T}^{N}\right)\right)^{d} \times\left(C^{\infty}\left(\mathbb{T}^{N}\right)\right)^{d}$, of (1.4)-(1.5) with $\theta^{i} \geq \theta_{0}>0$ for some constant $\theta_{0}$ that does not depend on $\epsilon$.

To prove Theorem 1.1, we establish the existence of solutions for (1.4)-(1.5) in Theorem 1.2 and prove $\epsilon$ independent bounds. The analysis of (1.4)-(1.5) begins in Section 3 where we examine various a priori estimates. Next, in Section 4, we consider separately the two different nonlinearities, $g(m)=\ln m$ and $g(m)=m^{\alpha}$. In these two sections, our estimates are uniform in $\epsilon$. In contrast, in Section 5, we prove $L^{\infty}$ estimates for $\theta$ and Lipschitz bounds for $u$ that depend on $\epsilon$. These are crucial in the proof of Theorem 1.2 that we present in Section 6. This proof combines the a priori estimates with the continuation method. The paper ends with the proof of Theorem 1.1 in Section 7 and a brief discussion of convergence and uniqueness in Section 8.

\section{Main ASSUmptions}

We begin by discussing the assumptions on $H^{i}, g$, and $\psi$ used in the study of (1.1)-(1.2). On the Hamiltonian, $H^{i}$, we assume standard hypotheses that hold in a large class of problems. In particular, they are satisfied by the example (1.3). To simplify the presentation, we select assumptions compatible with quadratic growth of the Hamiltonian, see Remark 2.3 below. Regarding the dependence on the measure: for every coordinate, $i$, we have the same nonlinearity, $g$, evaluated at the coordinate $\theta^{i}$. Some of our estimates are valid without substantial changes in the corresponding proofs if $g$ is replaced by a function, $g^{i}$, depending on all coordinates of $\theta$ or even on $x$. Naturally, Assumption 2 must be modified in a suitable way. Finally, we work with positive switching costs, $\psi^{i j}$. The positivity condition is natural in optimal switching because it prevents the occurrence of infinitely many switches. These conditions and the assumptions that follow are unlikely to give the most general case under which our techniques hold. Our choice reflects a balance between generality and simplicity of the proofs. 
Assumption 1. The Hamiltonian, $H^{i}$, the nonlinearity, $g$, and the switching cost, $\psi^{i j}$, satisfy:

(1) For $1 \leq i \leq d, H^{i}: \mathbb{T}^{N} \times \mathbb{R}^{N} \rightarrow \mathbb{R}$ is $C^{\infty}$ and positive.

(2) $g: \mathbb{R}^{+} \rightarrow \mathbb{R}$ is $C^{\infty}$ and strictly increasing; that is, $g^{\prime}>0$.

(3) For $1 \leq i, j \leq d$, the function $\psi^{i j}: \mathbb{T}^{N} \rightarrow \mathbb{R}$ is of class $C^{\infty}\left(\mathbb{T}^{N}\right)$. Furthermore, for $x \in \mathbb{T}^{N}, \psi^{i j}(x)>0$.

As usual, we identify whenever convenient, functions in $\mathbb{T}^{N}$ as $\mathbb{Z}^{N}$-periodic functions in $R^{N}$.

Assumption 2. The function $g$ satisfies the following.

(1) For any $C_{0}>0$, there exists $C_{1}$ such that

$$
\int_{\mathbb{T}^{N}} \theta g(\theta) d x \geq-C_{1}
$$

for any non-negative $\theta \in L^{1}\left(\mathbb{T}^{N}\right)$ with $\int_{\mathbb{T}^{N}} \theta d x \leq C_{0}$.

(2) There exists $C>0$ such that, for any $\theta>0$,

$$
g(\theta) \leq \frac{1}{2} \theta g(\theta)+C
$$

Remark 2.1. The functions $g(\theta)=\ln \theta$ and $g(\theta)=\theta^{\alpha}$, for $\alpha>0$, satisfy the preceding assumption.

Assumption 3. There exist constants, $c, C \geq 0$, such that

$$
H^{i}(p, x)-D_{p} H^{i}(p, x) \cdot p \leq-c H^{i}(p, x)+C
$$

for all $p \in \mathbb{R}^{N}, x \in \mathbb{T}^{N}$, and $1 \leq i \leq d$.

Remark 2.2. Consider the Lagrangian, $L^{i}$, associated with the Hamiltonian $H^{i}$ given by

$$
L^{i}(x, v)=\sup _{p \in \mathbb{R}^{N}}-p \cdot v-H^{i}(p, x)
$$

Because the supremum is achieved for $v=-D_{p} H^{i}(p, x)$,

$$
L^{i}(x, v)=D_{p} H^{i}(p, x) \cdot p-H^{i}(p, x) .
$$

Accordingly, the preceding hypothesis gives a lower bound on $L^{i}$.

Assumption 4. There exists $\gamma>0$ such that

$$
H_{p_{k} p_{j}}^{i}(p, x) \xi_{k} \xi_{j} \geq \gamma|\xi|^{2}
$$

for all $x \in \mathbb{T}^{N}$ and $p, \xi \in \mathbb{R}^{N}$.

There exist $C, c>0$ such that

$$
\begin{aligned}
& \left|D_{p p}^{2} H^{i}\right| \leq C, \\
& \left|D_{x p}^{2} H^{i}\right| \leq C(1+|p|), \\
& \left|D_{x x}^{2} H^{i}\right| \leq C\left(1+|p|^{2}\right) .
\end{aligned}
$$

Remark 2.3. The preceding assumption implies that there exists $C>0$ such that

$$
\frac{\gamma}{2}|p|^{2}-C \leq H^{i}(p, x) \leq C|p|^{2}+C,
$$


and

$$
\begin{aligned}
& \left|D_{p} H^{i}(p, x)\right| \leq C(1+|p|), \\
& \left|D_{x} H^{i}(p, x)\right| \leq C\left(1+|p|^{2}\right)
\end{aligned}
$$

for all $p \in \mathbb{R}^{N}$ and $x \in \mathbb{T}^{N}$.

Assumption 5. There exist constants, $C, \widetilde{C}>0$, and $\alpha \geq 0$ such that

$$
C \theta^{\alpha-1} \leq g^{\prime}(\theta) \leq \widetilde{C} \theta^{\alpha-1}+\widetilde{C}
$$

for any $\theta \geq 0$. Two specific cases of interest are

$\mathbf{L}-g(\theta)=\ln \theta$;

$\mathbf{P}-g(\theta)=\theta^{\alpha}, 0 \leq \alpha \leq 1$.

In the $\mathbf{P}$ case, the additional constraint, $\alpha<\frac{2}{N}$, is denoted by $\mathbf{P}-\frac{2}{N}$.

The next two assumptions are of a technical nature and are used in the study of the $\mathbf{P}-\frac{2}{N}$ case. Assumption 6 is employed in Proposition 4.3 to obtain a lower bound for $\theta^{i}$. Assumption 7 is fundamental in the proof of Proposition 5.1.

Assumption 6. For $1 \leq i \leq d$, we have

$$
D_{p x}^{2} H^{i}(0, x)=D_{x} H^{i}(0, x) D_{p} H^{i}(0, x)=0 \quad \text { for any } x \in \mathbb{T}^{N}
$$

and

$$
\max _{x \in \mathbb{T}^{N}} H^{i}(0, x)<1
$$

Remark 2.4. The preceding assumption is used to prove lower bounds for $\theta^{i}$. Because $H^{i} \geq 0$, the bound (2.10) gives an oscillation condition for $H^{i}(0, x)$. This oscillation condition is natural in light of the example considered in [28], Chapter 3. In that reference and also in [21], various examples of first-order MFGs are shown to have a vanishing density. The oscillation of $H(0, x)$ plays an essential role in these examples.

Remark 2.5. The number 1 on the right-hand side of (2.10) corresponds to the source term in the Fokker-Planck equation (1.5). Suppose that we modify (1.5) and consider a source, $v>0$; that is,

$$
-\operatorname{div}\left(D_{p} H^{i}\left(D u^{i}, x\right) \theta^{i}\right)+\theta^{i}+\sum_{j \neq i} \beta_{\epsilon}^{\prime}\left(u^{i}-u^{j}-\psi^{i j}\right) \theta^{i}-\beta_{\epsilon}^{\prime}\left(u^{j}-u^{i}-\psi^{j i}\right) \theta^{j}=v .
$$

Then, (2.10) becomes

$$
\max _{x \in \mathbb{T}^{N}} H^{i}(0, x)<v
$$

Assumption 7. The value $\alpha$ in Assumption 5 satisfies $\alpha \in\left[0, \alpha_{0}\right)$, where $\alpha_{0}$ solves

$$
2 \alpha_{0}=\left(\alpha_{0}+1\right) \beta(\beta-1), \quad \text { with } \beta=\sqrt{\frac{2^{*}}{2}}
$$

if $N>2$, and $\alpha_{0}=\infty$ if $N \leq 2$.

Remark 2.6. In the $N \leq 3$ case, the value $\alpha_{0}$ determined by (2.11) is larger than $\frac{2}{N}$. Whereas, if $N>3$ the opposite inequality holds.

Our last assumption is required in the study of the penalized problem. For $\epsilon>0$, we choose a penalty, $\beta_{\epsilon}$, satisfying the following assumption. 
Assumption 8. $\beta_{\epsilon}: \mathbb{R} \rightarrow \mathbb{R}$, smooth, with $\beta_{\epsilon}^{\prime} \geq 0, \beta_{\epsilon}^{\prime \prime} \geq 0$ with

$$
\beta_{\epsilon}(s)=0 \quad \text { for } s \leq 0, \quad \beta_{\epsilon}^{\prime}(s) \leq C \beta_{\epsilon}^{\prime \prime}(s) \quad \text { for } s>0,
$$

and $\beta_{\epsilon}(s) \rightarrow \infty$ as $\epsilon \rightarrow 0$ for $s>0$.

Remark 2.7. From the preceding assumption, we get

$$
\beta_{\epsilon}(s)-s \beta_{\epsilon}^{\prime}(s) \leq 0 \quad \text { for } s \in \mathbb{R} .
$$

The preceding assumption is standard in the setting of variational inequalities and optimal switching. In the context of MFGs, a similar penalty was used in [22] to study the obstacle problem.

\section{A PRIORI ESTIMATES}

Here, we prove a priori estimates for classical solutions of (1.4)-(1.5). The purpose of these estimates is twofold: first, to obtain the existence of solutions; second, to take the limit $\epsilon \rightarrow 0$. For that, we seek to prove bounds that are uniform in $\epsilon$. We begin with a simple consequence of the maximum principle for weakly coupled systems.

Proposition 3.1. Suppose that Assumptions 1 and 8 hold. Let $(u, \theta)$ be a $C^{\infty}$ solution of (1.4)-(1.5). Then, for $i=1, \ldots, d$, we have $\theta^{i} \geq 0$.

Proof. The proof of this Lemma is a straightforward application of the maximum principle to (1.5), see [5] for a similar proof.

As is standard in MFG problems, we can get several estimates by multiplying (1.4)-(1.5) by $1, \theta^{i}$ or $u^{i}$, adding or subtracting, and integrating by parts. We record these in the next lemma.

Lemma 3.2. Suppose that Assumptions $1-3$ and 8 hold. Let $(u, \theta)$ be a $C^{\infty}$ solution of (1.4)-(1.5). Then, there exists a constant, $C$, that does not depend on the particular solution nor on $\epsilon$, such that, for $i=1 \ldots d$,

$$
\begin{gathered}
0 \leq \int_{\mathbb{T}^{N}} \theta^{i} d x \leq C, \\
\left|\int_{\mathbb{T}^{N}} \theta^{i} g\left(\theta^{i}\right) d x\right| \leq C, \\
\left|\int_{\mathbb{T}^{N}} u^{i} d x\right| \leq C, \\
\int_{\mathbb{T}^{N}}\left|D u^{i}\right|^{2} \theta^{i} d x \leq C, \\
\sum_{i, j=1, i \neq j}^{d} \int_{\mathbb{T}^{N}} \beta_{\epsilon}^{\prime}\left(u^{i}-u^{j}-\psi^{i j}\right) \psi^{i j} \theta^{i} d x \leq C,
\end{gathered}
$$

and

$$
\int_{\mathbb{T}^{N}}\left|D u^{i}\right|^{2} d x \leq C
$$


Proof. By summing over $i$ the equations in (1.5), we gather that

$$
\sum_{i=1}^{d}-\operatorname{div}\left(D_{p} H^{i}\left(D u^{i}, x\right) \theta^{i}\right)+\theta^{i}=d .
$$

Hence, integrating on $\mathbb{T}^{N}$, we get

$$
\int_{\mathbb{T}^{N}} \theta^{i} d x \leq \sum_{i=1}^{d} \int_{\mathbb{T}^{N}} \theta^{i} d x=d
$$

for any $i=1, \ldots, d$. Thus, (3.1) holds. Due to Assumptions 1 and $8, H^{i}$ and $\beta_{\epsilon}$ are non-negative. Consequently, we infer that

$$
\int_{\mathbb{T}^{N}} u^{i} d x \leq \int_{\mathbb{T}^{N}} g\left(\theta^{i}\right) d x
$$

Next, we multiply (1.4) by $\theta^{i}$, sum over $i$, and integrate. Accordingly, we gather the identity

$$
\begin{aligned}
& \sum_{i=1}^{d} \int_{\mathbb{T}^{N}} H^{i}\left(D u^{i}, x\right) \theta^{i}+u^{i} \theta^{i}+\sum_{j \neq i} \beta_{\epsilon}\left(u^{i}-u^{j}-\psi^{i j}\right) \theta^{i} d x \\
& \quad=\sum_{i=1}^{d} \int_{\mathbb{T}^{N}} \theta^{i} g\left(\theta^{i}\right) d x .
\end{aligned}
$$

Next, we multiply (1.5) by $u^{i}$, add over $i$, and integrate by parts to conclude that

$$
\begin{aligned}
& \sum_{i=1}^{d} \int_{\mathbb{T}^{N}}\left[D_{p} H^{i}\left(D u^{i}, x\right) \cdot D u^{i} \theta^{i}+u^{i} \theta^{i}\right. \\
& \left.+\sum_{j \neq i} \beta_{\epsilon}^{\prime}\left(u^{i}-u^{j}-\psi^{i j}\right) \theta^{i} u^{i}-\beta_{\epsilon}^{\prime}\left(u^{j}-u^{i}-\psi^{j i}\right) \theta^{j} u^{i}\right] d x \\
& \quad=\sum_{i=1}^{d} \int_{\mathbb{T}^{N}} u^{i} d x .
\end{aligned}
$$

Subtracting equations (3.8) and (3.9), we get

$$
\begin{aligned}
& \sum_{i=1}^{d} \int_{\mathbb{T}^{N}} \theta^{i} g\left(\theta^{i}\right) d x \\
& =\sum_{i=1}^{d} \int_{\mathbb{T}^{N}} H^{i}\left(D u^{i}, x\right) \theta^{i}+u^{i} \theta^{i}+\sum_{j \neq i} \beta_{\epsilon}\left(u^{i}-u^{j}-\psi^{i j}\right) \theta^{i} d x \\
& =\sum_{i=1}^{d} \int_{\mathbb{T}^{N}}\left(H^{i}\left(D u^{i}, x\right)-D_{p} H^{i}\left(D u^{i}, x\right) \cdot D u^{i}\right) \theta^{i}+u^{i} d x \\
& +\sum_{i, j=1, i \neq j}^{d} \int_{\mathbb{T}^{N}} \beta_{\epsilon}\left(u^{i}-u^{j}-\psi^{i j}\right) \theta^{i} d x \\
& +\sum_{i, j=1, i \neq j}^{d} \int_{\mathbb{T}^{N}}-\beta_{\epsilon}^{\prime}\left(u^{i}-u^{j}-\psi^{i j}\right) \theta^{i} u^{i}+\beta_{\epsilon}^{\prime}\left(u^{j}-u^{i}-\psi^{j i}\right) \theta^{j} u^{i} d x .
\end{aligned}
$$


According to Assumption 3, we have

$$
\begin{aligned}
& \sum_{i=1}^{d} \int_{\mathbb{T}^{N}}\left(H^{i}\left(D u^{i}, x\right)-D_{p} H^{i}\left(D u^{i}, x\right) \cdot D u^{i}\right) \theta^{i} d x \\
& \quad \leq-c \sum_{i=1}^{d} \int_{\mathbb{T}^{N}} H^{i}\left(D u^{i}, x\right) \theta^{i} d x+C
\end{aligned}
$$

using (3.1). Moreover, we have

$$
\begin{aligned}
& \sum_{i, j=1, i \neq j}^{d} \int_{\mathbb{T}^{N}} \beta_{\epsilon}\left(u^{i}-u^{j}-\psi^{i j}\right) \theta^{i} d x \\
& +\sum_{i, j=1, i \neq j}^{d} \int_{\mathbb{T}^{N}}-\beta_{\epsilon}^{\prime}\left(u^{i}-u^{j}-\psi^{i j}\right) \theta^{i} u^{i}+\beta_{\epsilon}^{\prime}\left(u^{j}-u^{i}-\psi^{j i}\right) \theta^{j} u^{i} d x \\
= & \sum_{i, j=1, i \neq j}^{d} \int_{\mathbb{T}^{N}}\left[\beta_{\epsilon}\left(u^{i}-u^{j}-\psi^{i j}\right)-\beta_{\epsilon}^{\prime}\left(u^{i}-u^{j}-\psi^{i j}\right)\left(u^{i}-u^{j}-\psi^{i j}\right)\right] \theta^{i} d x \\
& -\sum_{i, j=1, i \neq j}^{d} \int_{\mathbb{T}^{N}} \beta_{\epsilon}^{\prime}\left(u^{i}-u^{j}-\psi^{i j}\right) \psi^{i j} \theta^{i} d x \\
\leq & -\sum_{i, j=1, i \neq j}^{d} \int_{\mathbb{T}^{N}} \beta_{\epsilon}^{\prime}\left(u^{i}-u^{j}-\psi^{i j}\right) \psi^{i j} \theta^{i} d x
\end{aligned}
$$

by (2.13) in Remark 2.7. Gathering the previous estimates, we conclude that

$$
\begin{aligned}
& \sum_{i=1}^{d} \int_{\mathbb{T}^{N}} \theta^{i} g\left(\theta^{i}\right)+c H^{i}\left(D u^{i}, x\right) \theta^{i} d x+\sum_{i, j=1, i \neq j}^{d} \int_{\mathbb{T}^{N}} \beta_{\epsilon}^{\prime}\left(u^{i}-u^{j}-\psi^{i j}\right) \psi^{i j} \theta^{i} d x \\
& \quad \leq \sum_{i=1}^{d} \int_{\mathbb{T}^{N}} u^{i} d x+C \leq \sum_{i=1}^{d} \int_{\mathbb{T}^{N}} g\left(\theta^{i}\right) d x+C
\end{aligned}
$$

using (3.7). Then, Assumption 2 implies

$$
\int_{\mathbb{T}^{N}} \theta^{i} g\left(\theta^{i}\right) d x \leq C
$$

On the other hand, (2.1) in Assumption 2 and (3.1) give

$$
\int_{\mathbb{T}^{N}} \theta^{i} g\left(\theta^{i}\right) d x \geq-C .
$$

Therefore, (3.2) holds. Using (3.2) and the bound (2.2) from Assumption 2, we get (3.3). In addition, for any $i=1, \ldots, d$,

$$
\int_{\mathbb{T}^{N}} H^{i}\left(D u^{i}, x\right) \theta^{i} d x \leq C .
$$

The last estimate combined with (2.4) implies (3.4). A similar argument yields $(3.5)$. 
Finally, the bound (3.6) follows from (1.4) by combining (2.4), the non-negativity of $\beta_{\epsilon}$, and the previous results with the estimate

$$
\int_{\mathbb{T}^{N}}\left|D u^{i}\right|^{2} d x \leq C+\int_{\mathbb{T}^{N}}\left(g\left(\theta^{i}\right)-u^{i}\right) d x \leq C .
$$

Lemma 3.3. Suppose that Assumptions $1-5$ and 8 hold. Let $(u, \theta)$ be a $C^{\infty}$ solution of (1.4)-(1.5). Then, there exists a constant, $C$, that does not depend on the particular solution nor on $\epsilon$, such that, for $i=1, \ldots, d$,

$$
\begin{gathered}
\int_{\mathbb{T}^{N}}\left|D^{2} u^{i}\right|^{2} \theta_{i} d x \leq C, \\
\int_{\mathbb{T}^{N}} g^{\prime}\left(\theta^{i}\right)\left|D \theta^{i}\right|^{2} d x \leq C,
\end{gathered}
$$

and

$$
\left\|\left(\theta^{i}\right)^{\frac{\alpha+1}{2}}\right\|_{W^{1,2}\left(\mathbb{T}^{N}\right)} \leq C
$$

Proof. We begin by differentiating (1.4) twice with respect to $x_{k}$ and then summing over $k$. In this way, we get

$$
\begin{aligned}
& D_{p} H^{i} \cdot D\left(\Delta u^{i}\right)+H_{x_{k} x_{k}}^{i}+2 H_{x_{k} p_{l}}^{i} u_{x_{l} x_{k}}^{i}+H_{p_{l} p_{m}}^{i} u_{x_{l} x_{k}}^{i} u_{x_{m} x_{k}}^{i}+\Delta u^{i} \\
& +\sum_{j \neq i} \beta_{\epsilon}^{\prime}\left(u^{i}-u^{j}-\psi^{i j}\right) \Delta\left(u^{i}-u^{j}-\psi^{i j}\right)+\beta_{\epsilon}^{\prime \prime}\left(u^{i}-u^{j}-\psi^{i j}\right)\left|D\left(u^{i}-u^{j}-\psi^{i j}\right)\right|^{2} \\
& \quad=\Delta\left(g\left(\theta^{i}\right)\right) .
\end{aligned}
$$

Next, we multiply the previous equation by $\theta^{i}$, add in the index $i$, and integrate by parts to conclude that

$$
\begin{aligned}
\sum_{i=1}^{d} \int_{\mathbb{T}^{N}} & \Delta\left(g\left(\theta^{i}\right)\right) \theta^{i} d x \\
= & \sum_{i=1}^{d} \int_{\mathbb{T}^{N}}\left(D_{p} H^{i} \cdot D\left(\Delta u^{i}\right)+\Delta u^{i}+\sum_{j \neq i} \beta_{\epsilon}^{\prime}\left(u^{i}-u^{j}-\psi^{i j}\right) \Delta u^{i}\right) \theta^{i} d x \\
& -\sum_{i=1}^{d} \sum_{j \neq i} \int_{\mathbb{T}^{N}} \beta_{\epsilon}^{\prime}\left(u^{i}-u^{j}-\psi^{i j}\right) \Delta\left(u^{j}+\psi^{i j}\right) \theta^{i} d x \\
& +\int_{\mathbb{T}^{N}}\left(H_{x_{k} x_{k}}^{i}+2 H_{x_{k} p_{l}}^{i} u_{x_{l} x_{k}}^{i}+H_{p_{l} p_{m}}^{i} u_{x_{l} x_{k}}^{i} u_{x_{m} x_{k}}^{i}\right) \theta^{i} d x \\
& +\sum_{i=1}^{d} \sum_{j \neq i} \int_{\mathbb{T}^{N}} \beta_{\epsilon}^{\prime \prime}\left(u^{i}-u^{j}-\psi^{i j}\right)\left|D\left(u^{i}-u^{j}-\psi^{i j}\right)\right|^{2} \theta^{i} d x .
\end{aligned}
$$


Multiplying (1.5) by $\Delta u^{i}$ and integrating by parts results in

$$
\begin{aligned}
& \sum_{i=1}^{d} \int_{\mathbb{T}^{N}}\left(D_{p} H^{i} \cdot D\left(\Delta u^{i}\right)+\Delta u^{i}+\sum_{j \neq i} \beta_{\epsilon}^{\prime}\left(u^{i}-u^{j}-\psi^{i j}\right) \Delta u^{i}\right) \theta^{i} d x \\
& =\sum_{i=1}^{d} \sum_{j \neq i} \int_{\mathbb{T}^{N}}\left(\beta_{\epsilon}^{\prime}\left(u^{j}-u^{i}-\psi^{j i}\right) \Delta u^{i} \theta^{j}+\Delta u^{i} d x\right. \\
& =\sum_{i=1}^{d} \sum_{j \neq i} \int_{\mathbb{T}^{N}}\left(\beta_{\epsilon}^{\prime}\left(u^{i}-u^{j}-\psi^{i j}\right) \Delta u^{j} \theta^{i} d x .\right.
\end{aligned}
$$

Using the previous identity in (3.14) gives

$$
\begin{aligned}
\sum_{i=1}^{d} \int_{\mathbb{T}^{N}} \Delta\left(g\left(\theta^{i}\right)\right) \theta^{i} d x & =-\sum_{i=1}^{d} \sum_{j \neq i} \int_{\mathbb{T}^{N}} \beta_{\epsilon}^{\prime}\left(u^{i}-u^{j}-\psi^{i j}\right) \Delta \psi^{i j} \theta^{i} d x \\
& +\int_{\mathbb{T}^{N}}\left(H_{x_{k} x_{k}}^{i}+2 H_{x_{k} p_{l}}^{i} u_{x_{l} x_{k}}^{i}+H_{p_{l} p_{m}}^{i} u_{x_{l} x_{k}}^{i} u_{x_{m} x_{k}}^{i}\right) \theta^{i} d x \\
& +\sum_{i=1}^{d} \sum_{j \neq i} \int_{\mathbb{T}^{N}} \beta_{\epsilon}^{\prime \prime}\left(u^{i}-u^{j}-\psi^{i j}\right)\left|D\left(u^{i}-u^{j}-\psi^{i j}\right)\right|^{2} \theta^{i} d x
\end{aligned}
$$

Taking into account that $\Delta \psi^{i j}$ is bounded and $\psi^{i j}>0$, estimate (3.5) implies that

$$
\left|\sum_{i, j=1, i \neq j}^{d} \int_{\mathbb{T}^{N}} \beta_{\epsilon}^{\prime}\left(u^{i}-u^{j}-\psi^{i j}\right) \Delta \psi^{i j} \theta^{i} d x\right| \leq C .
$$

Because $\beta_{\epsilon}^{\prime \prime} \geq 0$ and because of the uniform convexity from (2.4), (2.5) and (3.4), we obtain

$$
\begin{gathered}
\sum_{i=1}^{d} \int_{\mathbb{T}^{N}} g^{\prime}\left(\theta^{i}\right)\left|D \theta^{i}\right|^{2} d x+C \sum_{i=1}^{d} \int_{\mathbb{T}^{N}}\left|D^{2} u^{i}\right|^{2} \theta^{i} d x \\
\leq C \sum_{i=1}^{d} \int_{\mathbb{T}^{N}}\left(1+\left|D u^{i}\right|^{2}\right) \theta^{i} d x \leq C .
\end{gathered}
$$

Hence, we have (3.11) and (3.12). Moreover, from (3.12) and (2.8), we infer that

$$
\int_{\mathbb{T}^{N}}\left(\theta^{i}\right)^{\alpha-1}\left|D \theta^{i}\right|^{2} d x \leq C \int_{\mathbb{T}^{N}} g^{\prime}\left(\theta^{i}\right)\left|D \theta^{i}\right|^{2} d x \leq C ;
$$

that is, $\left|D\left(\theta^{i}\right)^{\frac{\alpha+1}{2}}\right| \in L^{2}\left(\mathbb{T}^{N}\right)$. By $(3.1), \theta^{i} \in L^{1}\left(\mathbb{T}^{N}\right)$. Thus, the previous inequality and the Poincaré inequality imply (3.13).

\section{Further a PRiori estimates}

In this section, we prove additional a priori estimates for logarithmic (Assumption $5 \mathbf{L}$ ) and power-like nonlinearities (Assumptions $5 \mathbf{P}$ and $\mathbf{P}-\frac{2}{N}$ ). These two cases are examined separately. Nevertheless, for both the logarithmic nonlinearity and for the power case, if $\alpha<\frac{2}{N}$ (Assumption $5 \mathbf{P}-\frac{2}{N}$ ), we obtain similar, $\epsilon$ independent lower bounds on $\theta$, on $\|u\|_{W^{2,2}\left(\mathbb{T}^{N}\right)}$, and on $\|u\|_{W^{1, p}\left(\mathbb{T}^{N}\right)}$ for any $p \geq 1$. 
4.1. The logarithmic case. Here, we consider the logarithmic nonlinearity $g(\theta)=$ $\ln \theta$.

Proposition 4.1. Suppose that Assumptions 1-4, $5 \mathbf{L}$ and 8 hold. Let $(u, \theta)$ be a $C^{\infty}$ solution of (1.4)-(1.5). Then, there exist constants $C, C_{p}, \theta_{0}>0$ that do not depend on the particular solution nor on $\epsilon$, such that, for $i=1, \ldots, d$ and for any $p \in[1,+\infty)$,

$$
\left\|u^{i}\right\|_{W^{1, p}\left(\mathbb{T}^{N}\right)} \leq C_{p}
$$

Moreover, for any $\gamma \in(0,1)$,

$$
\left\|u^{i}\right\|_{C^{\gamma}\left(\mathbb{T}^{N}\right)} \leq C
$$

In addition,

and

$$
\begin{gathered}
\theta^{i} \geq \theta_{0} \quad \text { in } \mathbb{T}^{N}, \\
\left\|\theta^{i}\right\|_{W^{1,2}\left(\mathbb{T}^{N}\right)} \leq C,
\end{gathered}
$$

Proof. In what follows, we use $C$ and $C_{p}$ to denote any of several constants, possibly depending on $p$ but independent of $\epsilon$. We remark that, for any $p \geq 1$, there exists a constant, $C_{p}>0$, such that

$$
\log \left(\theta^{i}\right) \leq\left(\theta^{i}\right)^{\frac{1}{p}}+C_{p}
$$

Therefore, from (1.4), using (2.6) in Remark 2.3 and the positivity of $\beta_{\epsilon}$, we infer that

$$
C\left|D u^{i}\right|^{2} \leq\left(\theta^{i}\right)^{\frac{1}{p}}+C_{p}-u^{i}=\left(\theta^{i}\right)^{\frac{1}{p}}+C_{p}-\left(u^{i}-\int_{\mathbb{T}^{N}} u^{i} d x\right)-\int_{\mathbb{T}^{N}} u^{i} d x .
$$

Combining the previous inequality with (3.3) yields

$$
\left|D u^{i}\right|^{2 p} \leq C \theta^{i}+C\left|u^{i}-\int_{\mathbb{T}^{N}} u^{i} d x\right|^{p}+C_{p} .
$$

Then, integrating, using (3.1) and the Poincaré inequality, we get

$$
\begin{aligned}
\int_{\mathbb{T}^{N}}\left|D u^{i}\right|^{2 p} d x & \leq C \int_{\mathbb{T}^{N}} \theta^{i} d x+C \int_{\mathbb{T}^{N}}\left|u^{i}-\int_{\mathbb{T}^{N}} u^{i} d x\right|^{p} d x+C_{p} \\
& \leq C_{p} \int_{\mathbb{T}^{N}}\left|D u^{i}\right|^{p} d x+C_{p} \\
& \leq \frac{1}{2} \int_{\mathbb{T}^{N}}\left|D u^{i}\right|^{2 p} d x+C_{p} .
\end{aligned}
$$

We conclude that, for any $p \geq 1$,

$$
\int_{\mathbb{T}^{N}}\left|D u^{i}\right|^{2 p} d x \leq C .
$$

This bound, together with (3.3) and the Poincaré inequality, gives (4.1). The Sobolev Embedding Theorem then implies (4.2). In particular, we have

$$
\left\|u^{i}\right\|_{L^{\infty}\left(\mathbb{T}^{N}\right)} \leq C .
$$


From (1.4), the previous estimate and the positivity of $H$ and $\beta$, we infer that

$$
\log \left(\theta^{i}\right) \geq-C
$$

from which (4.3) follows.

Estimate (4.4) is a consequence of (3.1), (3.12), (4.3) and the Poincaré inequality.

Finally, estimate (4.5) is a consequence of (3.3), (3.6), (3.11), (4.3) and the Poincaré inequality.

4.2. Power case. We devote this section to the study of power nonlinearities. We begin by examining the general case, Assumption $5 \mathbf{P}$. Then, we obtain additional results by considering Assumption $5 \mathbf{P}-\frac{2}{N}$. As in the previous section, our estimates are uniform in $\epsilon$.

Proposition 4.2. Suppose that Assumptions 1-4, $5 \mathbf{P}$ and 8 hold. Let $(u, \theta)$ be a $C^{\infty}$ solution of (1.4)-(1.5). Then, there exist constants, $C>0$ and $\gamma \in(0,1)$, that do not depend on the particular solution nor on $\epsilon$, such that, for $i=1, \ldots, d$,

$$
\left\|u^{i}\right\|_{W^{1, \frac{2}{\alpha}\left(\mathbb{T}^{N}\right)}} \leq C
$$

and

$$
\int_{\mathbb{T}^{N}}\left|D\left(\left(\theta^{i}\right)^{\frac{\alpha+1}{2}} D u^{i}\right)\right|^{\frac{2}{\alpha+1}} d x \leq C .
$$

If, in addition, Assumption $5 \mathbf{P}-\frac{2}{N}$ holds, then there exists $\gamma=\gamma(\alpha)$ such that

$$
\left\|u^{i}\right\|_{C^{\gamma}\left(\mathbb{T}^{N}\right)} \leq C \text {. }
$$

Proof. In what follows, we denote by $C$ several constants that are independent of $\epsilon$ and $\delta$. From (1.4), (2.6), and $\beta_{\epsilon} \geq 0$, we infer that

$$
C\left|D u^{i}\right|^{2} \leq\left(\theta^{i}\right)^{\alpha}+C-u^{i}=\left(\theta^{i}\right)^{\alpha}+C-\left(u^{i}-\int_{\mathbb{T}^{N}} u^{i} d x\right)-\int_{\mathbb{T}^{N}} u^{i} d x .
$$

Consequently, from (3.3),

$$
\left|D u^{i}\right|^{\frac{2}{\alpha}} \leq C \theta^{i}+C\left|u^{i}-\int_{\mathbb{T}^{N}} u^{i} d x\right|^{\frac{1}{\alpha}}+C .
$$

Then, integrating and using (3.1) and the Poincaré inequality, we get

$$
\begin{aligned}
\int_{\mathbb{T}^{N}}\left|D u^{i}\right|^{\frac{2}{\alpha}} d x & \leq C \int_{\mathbb{T}^{N}} \theta^{i} d x+C \int_{\mathbb{T}^{N}}\left|u^{i}-\int_{\mathbb{T}^{N}} u^{i} d x\right|^{\frac{1}{\alpha}} d x+C \\
& \leq C \int_{\mathbb{T}^{N}}\left|D u^{i}\right|^{\frac{1}{\alpha}} d x+C \\
& \leq \frac{1}{2} \int_{\mathbb{T}^{N}}\left|D u^{i}\right|^{\frac{2}{\alpha}} d x+C .
\end{aligned}
$$

Therefore,

$$
\int_{\mathbb{T}^{N}}\left|D u^{i}\right|^{\frac{2}{\alpha}} d x \leq C
$$

which gives, together with (3.3) and the Poincaré inequality, the bound (4.6). If $\alpha \in\left(0, \frac{2}{N}\right)$, then $\frac{2}{\alpha}>N$. Therefore, estimate (4.8) is a consequence of $(4.6)$ combined with the Sobolev Embedding Theorem. 
Next, to prove (4.7), we compute

$$
D\left(\left(\theta^{i}\right)^{\frac{\alpha+1}{2}} D u^{i}\right)=\frac{\alpha+1}{2} \theta^{\frac{\alpha-1}{2}} D u^{i} \otimes D \theta^{i}+\theta^{\frac{\alpha+1}{2}} D^{2} u^{i} .
$$

Now, using Hölder's inequality, we have

$$
\begin{aligned}
& \int_{\mathbb{T}^{N}}\left[\theta^{\frac{\alpha-1}{2}}\left|D \theta^{i}\right|\left|D u^{i}\right|\right]^{\frac{2}{\alpha+1}} d x \\
& =\int_{\mathbb{T}^{N}}\left(\theta^{i}\right)^{\frac{\alpha-1}{\alpha+1}}\left|D \theta^{i}\right|^{\frac{2}{\alpha+1}}\left|D u^{i}\right|^{\frac{2}{\alpha+1}} d x \\
& \leq\left[\int_{\mathbb{T}^{N}}\left[\left(\theta^{i}\right)^{\frac{\alpha-1}{\alpha+1}}\left|D \theta^{i}\right|^{\frac{2}{\alpha+1}}\right]^{\alpha+1} d x\right]^{\frac{1}{\alpha+1}}\left[\int_{\mathbb{T}^{N}}\left|D u^{i}\right|^{\frac{2}{\alpha+1}(\alpha+1)^{\prime}} d x\right]^{\frac{1}{(\alpha+1)^{\prime}}} \\
& =\left[\int_{\mathbb{T}^{N}}\left(\theta^{i}\right)^{\alpha-1}\left|D \theta^{i}\right|^{2} d x\right]^{\frac{1}{\alpha+1}}\left[\int_{\mathbb{T}^{N}}\left|D u^{i}\right|^{\frac{2}{\alpha}} d x\right]^{\frac{\alpha}{\alpha+1}} .
\end{aligned}
$$

From (3.13) and (4.6), we infer that

$$
\int_{\mathbb{T}^{N}}\left[\theta^{\frac{\alpha-1}{2}}\left|D \theta^{i}\right|\left|D u^{i}\right|\right]^{\frac{2}{\alpha+1}} d x \leq C .
$$

Next, using Hölder's inequality again, we have

$$
\begin{aligned}
\int_{\mathbb{T}^{N}}\left[\left|D^{2} u^{i}\right|\left(\theta^{i}\right)^{\frac{\alpha+1}{2}}\right]^{\frac{2}{\alpha+1}} d x & =\int_{\mathbb{T}^{N}}\left|D^{2} u^{i}\right|^{\frac{2}{\alpha+1}} \theta^{i} d x=\int_{\mathbb{T}^{N}}\left[\left|D^{2} u^{i}\right|^{2} \theta^{i}\right]^{\frac{1}{\alpha+1}}\left(\theta^{i}\right)^{\frac{\alpha}{\alpha+1}} d x \\
& \leq\left[\int_{\mathbb{T}^{N}}\left|D^{2} u^{i}\right|^{2} \theta^{i} d x\right]^{\frac{1}{\alpha+1}}\left[\int_{\mathbb{T}^{N}} \theta^{i} d x\right]^{\frac{\alpha}{\alpha+1}} .
\end{aligned}
$$

From (3.1) and (3.11), we gather the bound

$$
\int_{\mathbb{T}^{N}}\left[\left|D^{2} u^{i}\right|\left(\theta^{i}\right)^{\frac{\alpha+1}{2}}\right]^{\frac{2}{\alpha+1}} d x \leq C .
$$

Estimate (4.7) is then a consequence of (4.9), (4.10) and (4.11).

Proposition 4.3. Suppose that Assumptions 1-4, $5 \mathbf{P}-\frac{2}{N}$, 6, and 8 hold. Let $(u, \theta)$ be a $C^{\infty}$ solution of (1.4)-(1.5). Then, for $i=1, \ldots, d$ and any $x \in \mathbb{T}^{N}$, we have

$$
\theta^{i}(x) \geq\left(1-\max _{\substack{x \in \mathbb{T}^{N} \\ j=1, \ldots, d}} H^{j}(0, x)\right)^{\frac{1}{\alpha}} .
$$

Moreover, there exists $C>0$ that does not depend on the particular solution nor on $\epsilon$, such that

$$
\left\|\theta^{i}\right\|_{W^{1,2}\left(\mathbb{T}^{N}\right)} \leq C
$$

and

$$
\left\|u^{i}\right\|_{W^{2,2}\left(\mathbb{T}^{N}\right)} \leq C
$$

Proof. We begin the proof by establishing a lower bound on $u$. Let $i \in\{1, \ldots, d\}$ and $x_{0} \in \mathbb{T}^{N}$ be such that

$$
u^{i}\left(x_{0}\right)=\min _{\substack{j=1, \ldots, d \\ x \in \mathbb{N} N}} u^{j}(x) .
$$


Then, we have

$$
D u^{i}\left(x_{0}\right)=0, D^{2} u^{i}\left(x_{0}\right) \geq 0
$$

and

$$
u^{i}\left(x_{0}\right) \leq u^{j}\left(x_{0}\right) \text { for any } j=1, \ldots, d .
$$

In particular, the last inequality implies

$\beta_{\epsilon}\left(u^{i}\left(x_{0}\right)-u^{j}\left(x_{0}\right)-\psi^{i j}\left(x_{0}\right)\right)=\beta_{\epsilon}^{\prime}\left(u^{i}\left(x_{0}\right)-u^{j}\left(x_{0}\right)-\psi^{i j}\left(x_{0}\right)\right)=0 \quad$ for any $j=1, \ldots, d$.

From (1.4), (4.15), and (4.16), we infer that

$$
H^{i}\left(0, x_{0}\right)+u^{i}\left(x_{0}\right)=g\left(\theta^{i}\left(x_{0}\right)\right) .
$$

We can substitute

$$
\theta^{i}=g^{-1}\left(H^{i}\left(D u^{i}, x\right)+u^{i}+\sum_{j \neq i} \beta_{\epsilon}\left(u^{i}-u^{j}-\psi^{i j}\right)\right)
$$

in (1.5) to get

$$
\begin{aligned}
& -\theta^{i} H_{p_{k} p_{j}}^{i} u_{x_{j} x_{k}}^{i}-\theta^{i} H_{p_{k} x_{k}}^{i}-\frac{1}{g^{\prime}\left(\theta^{i}\right)}\left[H_{p_{k}}^{i} H_{p_{j}}^{i} u_{x_{j} x_{k}}^{i}+H_{x_{k}}^{i} H_{p_{k}}^{i}+H_{p_{k}}^{i} u_{x_{k}}^{i}\right. \\
& \left.+\sum_{j \neq i} \beta_{\epsilon}^{\prime}\left(u^{i}-u^{j}-\psi^{i j}\right)\left(u^{i}-u^{j}-\psi^{i j}\right)_{x_{k}} H_{p_{k}}^{i}\right]+\theta^{i}+\sum_{j \neq i} \beta_{\epsilon}^{\prime}\left(u^{i}-u^{j}-\psi^{i j}\right) \theta^{i} \\
& =\sum_{j \neq i} \beta_{\epsilon}^{\prime}\left(u^{j}-u^{i}-\psi^{j i}\right) \theta^{j}+1 .
\end{aligned}
$$

Evaluating at $x=x_{0}$ and using (2.4), (4.15) and (4.16), we obtain

$$
-\theta^{i} H_{p_{k} x_{k}}^{i}\left(0, x_{0}\right)-\frac{1}{g^{\prime}\left(\theta^{i}\left(x_{0}\right)\right)} H_{x_{k}}^{i}\left(0, x_{0}\right) H_{p_{k}}^{i}\left(0, x_{0}\right)+\theta^{i}\left(x_{0}\right) \geq 1 \text {. }
$$

Since $H^{i}$ satisfies $(2.9)$, the preceding inequality can be rewritten as

$$
\theta^{i}\left(x_{0}\right) \geq 1
$$

Then, (4.17) and the last estimate imply

$$
u^{i}\left(x_{0}\right) \geq g^{-1}\left(1-H^{i}\left(0, x_{0}\right)\right) .
$$

Now, from (1.4), (4.18), (2.9), (2.10) and the positivity of $H^{j}$ and $\beta_{\epsilon}$, we infer that, for any $x \in \mathbb{T}^{N}$ and $j=1, \ldots, d$,

$$
g\left(\theta^{j}(x)\right) \geq H^{j}\left(D u^{j}, x\right)+u^{j}(x) \geq u^{i}\left(x_{0}\right) \geq 1-H^{i}\left(0, x_{0}\right) .
$$

Thus, (4.12) follows from the preceding inequality.

Estimate (4.13) follows by combining (3.1), (3.12), (4.12) and the Poincaré inequality. Finally, (4.14) is a consequence of $(3.3),(3.6),(3.11),(4.12)$, and the Poincaré inequality. 


\section{LIPSCHITZ BOUNDS}

In this section, we prove the Lipschitz continuity of $u$ for any solution $(u, \theta)$ of (1.4)-(1.5). These bounds are used to establish the existence of solutions by the continuation method. In contrast to the results in the preceding sections, the estimates here depend on $\epsilon$ and are not valid for (1.1)-(1.2).

Lemma 5.1. Suppose that Assumptions 1-5 and 8 hold, and that either

- Assumption $5 \mathbf{L}$ or

- Assumptions 5 P- $\frac{2}{N}, 6$ and 7

hold. Let $(u, \theta)$ be a $C^{\infty}$ solution of (1.4)-(1.5). Then, there exists $C_{\epsilon}>0$ that does not depend on the particular solution, such that, for any $i=1, \ldots, d$,

$$
\left\|\theta^{i}\right\|_{L^{\infty}\left(\mathbb{T}^{N}\right)} \leq C_{\epsilon}
$$

and

$$
\left\|u^{i}\right\|_{W^{1, \infty}\left(\mathbb{T}^{N}\right)} \leq C_{\epsilon} .
$$

Proof. First, note that (5.2) is an immediate consequence of (5.1). Indeed, by combining (5.1) with (2.6), by the positivity of $\beta_{\epsilon}$, by the boundedness of $u^{i}$ (c.f. Proposition 4.1 and Proposition 4.2), and by (1.4), we get

$$
C\left|D u^{i}\right|^{2} \leq H^{i}\left(D u^{i}\right)+C \leq g\left(\theta^{i}\right)-u^{i}+C \leq C
$$

Consequently, we only need to prove (5.1).

If Assumption $5 \mathbf{L}$ holds or if Assumptions $5 \mathbf{P}-\frac{2}{N}$ and 6 hold by, respectively, Propositions 4.1 and 4.3 , then there exists $\theta_{0}>0$, such that, for any $i=1, \ldots, d$ and for any $x \in \mathbb{T}^{N}$,

$$
\theta^{i}(x) \geq \theta_{0}>0
$$

For any fixed $\epsilon$, by (4.2) and (4.8), there exists a constant, $C$, depending on $\epsilon$, such that, for any $i, j=1, \ldots, d$,

$$
\beta_{\epsilon}^{\prime}\left(u^{i}-u^{j}-\psi^{i j}\right) \leq C
$$

To prove (5.1), we use a technique introduced in [16] and used in [22] to study a mean-field-game obstacle problem. For $p>0$, we multiply the equation (1.5) by 
$\operatorname{div}\left(\left(\theta^{i}\right)^{p} D_{p} H^{i}\left(D u^{i}, x\right)\right)$ and integrate by parts. Accordingly, we get

$$
\begin{aligned}
& \int_{\mathbb{T}^{N}}\left[\theta^{i}+\sum_{j \neq i} \beta_{\epsilon}^{\prime}\left(u^{i}-u^{j}-\psi^{i j}\right) \theta^{i}-\sum_{j \neq i} \beta_{\epsilon}^{\prime}\left(u^{j}-u^{i}-\psi^{j i}\right) \theta^{j}\right] \operatorname{div}\left(\left(\theta^{i}\right)^{p} D_{p} H^{i}\right) d x \\
& =\int_{\mathbb{T}^{N}}\left(\theta^{i} H_{p_{k}}^{i}\right)_{x_{k}}\left(\left(\theta^{i}\right)^{p} H_{p_{j}}^{i}\right)_{x_{j}} d x \\
& =\int_{\mathbb{T}^{N}}\left(\theta^{i} H_{p_{k}}^{i}\right)_{x_{j}}\left(\left(\theta^{i}\right)^{p} H_{p_{j}}^{i}\right)_{x_{k}} d x \\
& =\int_{\mathbb{T}^{N}}\left(\theta^{i}\left(H_{p_{k}}^{i}\right)_{x_{j}}+\theta_{x_{j}}^{i} H_{p_{k}}^{i}\right)\left(\left(\theta^{i}\right)^{p}\left(H_{p_{j}}^{i}\right)_{x_{k}}+p\left(\theta^{i}\right)^{p-1} \theta_{x_{k}}^{i} H_{p_{j}}^{i}\right) d x \\
& =\int_{\mathbb{T}^{N}}\left(\theta^{i}\right)^{p+1}\left(H_{p_{k}}^{i}\right)_{x_{j}}\left(H_{p_{j}}^{i}\right)_{x_{k}}+p\left(\theta^{i}\right)^{p-1} H_{p_{k}}^{i} \theta_{x_{k}}^{i} H_{p_{j}}^{i} \theta_{x_{j}}^{i} \\
& +(p+1)\left(\theta^{i}\right)^{p} \theta_{x_{k}}^{i} H_{p_{j}}^{i}\left(H_{p_{k}}^{i}\right)_{x_{j}} d x \\
& =: \int_{\mathbb{T}^{N}} I_{1}+I_{2}+I_{3} d x .
\end{aligned}
$$

Using (2.5) in Assumption 4, we get

$$
\begin{aligned}
I_{1} & =\left(\theta^{i}\right)^{p+1}\left(H_{p_{k} p_{l}}^{i} u_{x_{l} x_{j}}+H_{p_{k} x_{j}}^{i}\right)\left(H_{p_{j} p_{m}}^{i} u_{x_{m} x_{k}}+H_{p_{j} x_{k}}^{i}\right) \\
& \geq\left(\theta^{i}\right)^{p+1}\left[\gamma^{2}\left|D^{2} u^{i}\right|^{2}-C\left(1+\left|D u^{i}\right|\right)\left|D^{2} u^{i}\right|-C\left(1+\left|D u^{i}\right|^{2}\right)\right] \\
& \geq\left(\theta^{i}\right)^{p+1} \tilde{\gamma}^{2}\left|D^{2} u^{i}\right|^{2}-C\left(\theta^{i}\right)^{p+1}\left(1+\left|D u^{i}\right|^{2}\right)
\end{aligned}
$$

for some $\tilde{\gamma}>0$. Clearly,

$$
I_{2}=p\left(\theta^{i}\right)^{p-1}\left|D_{p} H^{i} \cdot D \theta^{i}\right|^{2} .
$$

Next, we estimate $I_{3}$ from below. From equation (1.4), we gather that

$$
H_{p_{j}}^{i} u_{x_{j} x_{l}}^{i}=g^{\prime}\left(\theta^{i}\right) \theta_{x_{l}}^{i}-H_{x_{l}}^{i}-u_{x_{l}}^{i}-\sum_{j \neq i} \beta_{\epsilon}^{\prime}\left(u^{i}-u^{j}-\psi^{i j}\right)\left(u^{i}-u^{j}-\psi^{i j}\right)_{x_{l}}
$$

The estimate (2.8) in Assumption 4 and the lower bound (5.3) on $\theta^{i}$ imply the existence of a positive constant, $C_{0}$ (depending on $\epsilon$ ), such that

$$
g^{\prime}\left(\theta^{i}\right) \theta^{i} \geq C_{0}>0
$$

Then, using (2.4), (2.5), (5.4), (5.6), (5.7), and the Cauchy-Schwarz inequality, we get

$$
\begin{aligned}
I_{3} & =(p+1)\left(\theta^{i}\right)^{p} \theta_{x_{k}}^{i} H_{p_{j}}^{i}\left(H_{p_{k} p_{l}}^{i} u_{x_{l} x_{j}}^{i}+H_{p_{k} x_{j}}^{i}\right) \\
& =(p+1) g^{\prime}\left(\theta^{i}\right)\left(\theta^{i}\right)^{p} H_{p_{k} p_{l}}^{i} \theta_{x_{k}}^{i} \theta_{x_{l}}^{i}+(p+1)\left(\theta^{i}\right)^{p} \theta_{x_{k}}^{i}\left(H_{p_{j}}^{i} H_{p_{k} x_{j}}^{i}-H_{p_{k} p_{l}}^{i} H_{x_{l}}^{i}\right) \\
& -(p+1)\left(\theta^{i}\right)^{p} H_{p_{k} p_{l}}^{i} \theta_{x_{k}}^{i}\left(u_{x_{l}}^{i}+\sum_{j \neq i} \beta_{\epsilon}^{\prime}\left(u^{i}-u^{j}-\psi^{i j}\right)\left(u^{i}-u^{j}-\psi^{i j}\right)_{x_{l}}\right) \\
& \geq(p+1) \gamma C_{0}\left(\theta^{i}\right)^{p-1}\left|D \theta^{i}\right|^{2}-C(p+1)\left(\theta^{i}\right)^{p}\left|D \theta^{i}\right|\left(1+\left|D u^{i}\right|^{2}\right) \\
& -C(p+1)\left(\theta^{i}\right)^{p}\left|D \theta^{i}\right|\left(1+\left|D u^{i}\right|\right)-\sum_{j \neq i} C(p+1)\left(\theta^{i}\right)^{p}\left|D \theta^{i}\right|\left(1+\left|D u^{j}\right|\right) \\
& \geq C(p+1)\left(\theta^{i}\right)^{p-1}\left|D \theta^{i}\right|^{2}-C(p+1)\left(\theta^{i}\right)^{p+1}\left(1+\left|D u^{i}\right|^{4}+\sum_{j \neq i}\left|D u^{j}\right|^{2}\right) .
\end{aligned}
$$


Next, we bound the left-hand side of (5.5) from above. Using (2.5), (5.4), and the Cauchy-Schwarz inequality, we obtain

$$
\begin{aligned}
& \left(\theta^{i}+\sum_{j \neq i} \beta_{\epsilon}^{\prime}\left(u^{i}-u^{j}-\psi^{i j}\right) \theta^{i}\right) \operatorname{div}\left(\left(\theta^{i}\right)^{p} D_{p} H^{i}\right) \\
& =\left[\theta^{i}+\sum_{j \neq i} \beta_{\epsilon}^{\prime}\left(u^{i}-u^{j}-\psi^{i j}\right) \theta^{i}\right]\left[p\left(\theta^{i}\right)^{p-1} D_{p} H^{i} \cdot D \theta^{i}+\left(\theta^{i}\right)^{p} H_{p_{k} p_{j}}^{i} u_{x_{j} x_{k}}^{i}+\left(\theta^{i}\right)^{p} H_{p_{k} x_{k}}^{i}\right] \\
& \leq C \theta^{i}\left[p\left(\theta^{i}\right)^{p-1}\left|D_{p} H^{i} \cdot D \theta^{i}\right|+\left(\theta^{i}\right)^{p}\left|H_{p_{k} p_{j}}^{i} u_{x_{j} x_{k}}^{i}\right|+\left(\theta^{i}\right)^{p}\left|H_{p_{k} x_{k}}^{i}\right|\right] \\
& \leq \frac{p}{2}\left(\theta^{i}\right)^{p-1}\left|D_{p} H^{i} \cdot D \theta^{i}\right|^{2}+\frac{\tilde{\gamma}^{2}}{2}\left(\theta^{i}\right)^{p+1}\left|D^{2} u^{i}\right|^{2}+C p\left(\theta^{i}\right)^{p+1}\left(1+\left|D u^{i}\right|\right) .
\end{aligned}
$$

Similarly,

$$
\begin{aligned}
& -\sum_{j \neq i} \beta_{\epsilon}^{\prime}\left(u^{j}-u^{i}-\psi^{j i}\right) \theta^{j} \operatorname{div}\left(\left(\theta^{i}\right)^{p} D_{p} H^{i}\right) \\
& \leq \sum_{j \neq i} C \theta^{j}\left|p\left(\theta^{i}\right)^{p-1}\right| D_{p} H^{i} \cdot D \theta^{i}\left|+\left(\theta^{i}\right)^{p}\right| H_{p_{k} p_{j}}^{i} u_{x_{j} x_{k}}^{i}\left|+\left(\theta^{i}\right)^{p}\right| H_{p_{k} x_{k}}^{i}|| \\
& \leq \sum_{j \neq i} C \theta^{j}\left[p\left(\theta^{i}\right)^{\frac{p-1}{2}}\left(\theta^{i}\right)^{\frac{p-1}{2}}\left|D_{p} H^{i} \cdot D \theta^{i}\right|+\left(\theta^{i}\right)^{\frac{p-1}{2}}\left(\theta^{i}\right)^{\frac{p+1}{2}}\left|H_{p_{k} p_{j}}^{i} u_{x_{j} x_{k}}^{i}\right|+\left(\theta^{i}\right)^{\frac{p-1}{2}}\left(\theta^{i}\right)^{\frac{p+1}{2}}\left|H_{p_{k} x_{k}}^{i}\right|\right] \\
& \leq \sum_{j \neq i} C p\left(\theta^{j}\right)^{2}\left(\theta^{i}\right)^{p-1}+\frac{p}{2}\left(\theta^{i}\right)^{p-1}\left|D_{p} H^{i} \cdot D \theta^{i}\right|^{2}+\frac{\tilde{\gamma}^{2}}{2}\left(\theta^{i}\right)^{p+1}\left|D^{2} u^{i}\right|^{2}+C p\left(\theta^{i}\right)^{p+1}\left(1+\left|D u^{i}\right|^{2}\right) .
\end{aligned}
$$

From the preceding estimates, we conclude that

$$
\begin{aligned}
& C(p+1) \int_{\mathbb{T}^{N}}\left(\theta^{i}\right)^{p-1}\left|D \theta^{i}\right|^{2} d x-C(p+1) \int_{\mathbb{T}^{N}}\left(\theta^{i}\right)^{p+1}\left(1+\left|D u^{i}\right|^{4}+\sum_{j \neq i}\left|D u^{j}\right|^{2}\right) d x \\
& +p \int_{\mathbb{T}^{N}}\left(\theta^{i}\right)^{p-1}\left|D_{p} H^{i} \cdot D \theta^{i}\right|^{2} d x \\
& +\tilde{\gamma}^{2} \int_{\mathbb{T}^{N}}\left(\theta^{i}\right)^{p+1}\left|D^{2} u^{i}\right|^{2}-C \int_{\mathbb{T}^{N}}\left(\theta^{i}\right)^{p+1}\left(1+\left|D u^{i}\right|^{2}\right) d x \\
& \leq \int_{\mathbb{T}^{N}} I_{1}+I_{2}+I_{3} d x \\
& =\int_{\mathbb{T}^{N}}\left[\theta^{i}+\sum_{j \neq i} \beta_{\epsilon}^{\prime}\left(u^{i}-u^{j}-\psi^{i j}\right) \theta^{i}-\sum_{j \neq i} \beta_{\epsilon}^{\prime}\left(u^{j}-u^{i}-\psi^{j i}\right) \theta^{j}\right] \operatorname{div}\left(\left(\theta^{i}\right)^{p} D_{p} H^{i}\right) d x \\
& \leq p \int_{\mathbb{T}^{N}}\left(\theta^{i}\right)^{p-1}\left|D_{p} H^{i} \cdot D \theta^{i}\right|^{2} d x+\tilde{\gamma}^{2} \int_{\mathbb{T}^{N}}\left(\theta^{i}\right)^{p+1}\left|D^{2} u^{i}\right|^{2} d x \\
& +C p \int_{\mathbb{T}^{N}}\left(\theta^{i}\right)^{p+1}\left(1+\left|D u^{i}\right|^{2}\right) d x+\sum_{j \neq i} C p \int_{\mathbb{T}^{N}}\left(\theta^{j}\right)^{2}\left(\theta^{i}\right)^{p-1} .
\end{aligned}
$$

Consequently,

$\int_{\mathbb{T}^{N}}\left(\theta^{i}\right)^{p-1}\left|D \theta^{i}\right|^{2} d x \leq \int_{\mathbb{T}^{N}}\left(\theta^{i}\right)^{p+1}\left(1+\left|D u^{i}\right|^{4}+\sum_{j \neq i}\left|D u^{j}\right|^{2}\right) d x+\sum_{j \neq i} C \int_{\mathbb{T}^{N}}\left(\theta^{j}\right)^{2}\left(\theta^{i}\right)^{p-1} d x$.

Applying Young's inequality, we gather

$$
\left(\theta^{j}\right)^{2}\left(\theta^{i}\right)^{p-1} \leq \frac{2}{p+1}\left(\theta^{j}\right)^{p+1}+\frac{p-1}{p+1}\left(\theta^{i}\right)^{p+1} .
$$


Therefore,

$\int_{\mathbb{T}^{N}}\left(\theta^{i}\right)^{p-1}\left|D \theta^{i}\right|^{2} d x \leq \int_{\mathbb{T}^{N}}\left(\theta^{i}\right)^{p+1}\left(1+\left|D u^{i}\right|^{4}+\sum_{j \neq i}\left|D u^{j}\right|^{2}\right) d x+\sum_{j \neq i} C \int_{\mathbb{T}^{N}}\left(\theta^{j}\right)^{p+1} d x$.

If $N=1,(5.1)$ is a consequence of Morrey's Theorem. If $N=2$, then $\theta^{i} \in L^{p}$ for all $p$. Moreover, in this case, the argument that follows can be modified by replacing the Sobolev exponent, $2^{*}$, by any arbitrarily large number, $M$. Therefore, we assume that $N>2$. Accordingly, by (3.13), we have $\theta^{i} \in L^{\frac{2^{*}(1+\alpha)}{2}}$. In addition, Sobolev's inequality provides the bound

$$
\begin{aligned}
\left(\int_{\mathbb{T}^{N}}\left(\theta^{i}\right)^{\frac{p+1}{2} 2^{*}} d x\right)^{\frac{2}{2^{*}}} & \leq C \int_{\mathbb{T}^{N}}\left(\theta^{i}\right)^{p+1} d x+C \int_{\mathbb{T}^{N}}\left|D\left(\left(\theta^{i}\right)^{\frac{p+1}{2}}\right)\right|^{2} d x \\
& =C \int_{\mathbb{T}^{N}}\left(\theta^{i}\right)^{p+1} d x+C(p+1)^{2} \int_{\mathbb{T}^{N}}\left(\theta^{i}\right)^{p-1}\left|D \theta^{i}\right|^{2} d x
\end{aligned}
$$

Let $\beta:=\sqrt{\frac{2^{*}}{2}}=\sqrt{\frac{N}{N-2}}>1$. Consider first the $\mathbf{P}$ case. Then, Assumption 7 implies that

$$
2 \alpha \leq(\alpha+1) \beta^{2} \frac{\beta-1}{\beta} .
$$

The previous inequality together with (2.6) implies that

$$
\left|D u^{i}\right|^{4} \leq C\left(g\left(\theta^{i}\right)\right)^{2}+C \leq C\left(\theta^{i}\right)^{2 \alpha}+C \leq C\left(1+\left(\theta^{i}\right)^{(\alpha+1) \beta^{2} \frac{\beta-1}{\beta}}\right) .
$$

The same inequality holds in the logarithmic case with $\alpha=0$ :

$$
\left|D u^{i}\right|^{4} \leq C\left(g\left(\theta^{i}\right)\right)^{2}+C \leq C\left(\log \left(\theta^{i}\right)\right)^{2}+C \leq C\left(1+\left(\theta^{i}\right)^{\beta^{2} \frac{\beta-1}{\beta}}\right) .
$$

Therefore, from Hölder's inequality, we get

$$
\begin{aligned}
& \int_{\mathbb{T}^{N}}\left(\theta^{i}\right)^{p+1}\left(1+\left|D u^{i}\right|^{4}\right) d x \leq C \int_{\mathbb{T}^{N}}\left(\theta^{i}\right)^{p+1}\left(1+\left(\theta^{i}\right)^{(\alpha+1) \beta^{2} \frac{\beta-1}{\beta}}\right) d x \\
& \leq C \int_{\mathbb{T}^{N}}\left(\theta^{i}\right)^{p+1} d x+C\left(\int_{\mathbb{T}^{N}}\left(\theta^{i}\right)^{(p+1) \beta} d x\right)^{\frac{1}{\beta}}\left(\int_{\mathbb{T}^{N}}\left(\theta^{i}\right)^{(\alpha+1) \beta^{2}} d x\right)^{\frac{\beta-1}{\beta}} \\
& \leq C \int_{\mathbb{T}^{N}}\left(\theta^{i}\right)^{p+1} d x+C\left(\int_{\mathbb{T}^{N}}\left(\theta^{i}\right)^{(p+1) \beta} d x\right)^{\frac{1}{\beta}} \\
& \leq C\left(\int_{\mathbb{T}^{N}}\left(\theta^{i}\right)^{(p+1) \beta} d x\right)^{\frac{1}{\beta}} .
\end{aligned}
$$

Similarly,

$$
\begin{aligned}
& \int_{\mathbb{T}^{N}}\left(\theta^{i}\right)^{p+1}\left(1+\left|D u^{j}\right|^{2}\right) d x \leq C \int_{\mathbb{T}^{N}}\left(\theta^{i}\right)^{p+1}\left(1+\left(\theta^{j}\right)^{(\alpha+1) \beta^{2} \frac{\beta-1}{\beta}}\right) d x \\
& \leq C \int_{\mathbb{T}^{N}}\left(\theta^{i}\right)^{p+1} d x+C\left(\int_{\mathbb{T}^{N}}\left(\theta^{i}\right)^{(p+1) \beta} d x\right)^{\frac{1}{\beta}}\left(\int_{\mathbb{T}^{N}}\left(\theta^{j}\right)^{(\alpha+1) \beta^{2}} d x\right)^{\frac{\beta-1}{\beta}} \\
& \leq C \int_{\mathbb{T}^{N}}\left(\theta^{i}\right)^{p+1} d x+C\left(\int_{\mathbb{T}^{N}}\left(\theta^{i}\right)^{(p+1) \beta} d x\right)^{\frac{1}{\beta}} \\
& \leq C\left(\int_{\mathbb{T}^{N}}\left(\theta^{i}\right)^{(p+1) \beta} d x\right)^{\frac{1}{\beta}} .
\end{aligned}
$$


The last two inequalities, combined with (5.8) and (5.9) give the bound

$$
\left(\int_{\mathbb{T}^{N}}\left(\theta^{i}\right)^{(p+1) \beta^{2}} d x\right)^{\frac{1}{\beta^{2}}} \leq C p^{2}\left(\int_{\mathbb{T}^{N}}\left(\theta^{i}\right)^{(p+1) \beta} d x\right)^{\frac{1}{\beta}}+C p^{2} \sum_{j \neq i}\left(\int_{\mathbb{T}^{N}}\left(\theta^{j}\right)^{(p+1) \beta} d x\right)^{\frac{1}{\beta}}
$$

for $i=1, \ldots, d$. Summing on $i$, we finally obtain

$$
\sum_{i=1}^{d}\left(\int_{\mathbb{T}^{N}}\left(\theta^{i}\right)^{(p+1) \beta^{2}} d x\right)^{\frac{1}{\beta^{2}}} \leq C p^{2} \sum_{i=1}^{d}\left(\int_{\mathbb{T}^{N}}\left(\theta^{i}\right)^{(p+1) \beta} d x\right)^{\frac{1}{\beta}} .
$$

Arguing as in [16], we get

$$
\sum_{i=1}^{d}\left\|\theta^{i}\right\|_{L^{\infty}\left(\mathbb{T}^{N}\right)} \leq C
$$

and, hence, (5.1).

Corollary 5.2. Suppose that Assumptions 1-5 and 8 hold, and either

- Assumption $5 \mathbf{L}$ or

- Assumptions 5 P- $\frac{2}{N}, 6$ and 7

hold. Let $(u, \theta)$ be a $C^{\infty}$ solution of (1.4)-(1.5). Then, for any $k \in \mathbb{N}$, there exists $C_{\epsilon, k}>0$ that does not depend on the particular solution, such that

$$
\left\|u^{i}\right\|_{W^{k, \infty}\left(\mathbb{T}^{N}\right)}+\left\|\theta^{i}\right\|_{W^{k, \infty}\left(\mathbb{T}^{N}\right)} \leq C_{\epsilon, k},
$$

for any $i=1, \ldots, d$.

Proof. If Assumption $5 \mathbf{L}$ holds or if Assumptions $5 \mathbf{P}-\frac{2}{N}$ and 6 hold by, respectively, Propositions 4.1, and 4.3, then there exists $\theta_{0}>0$, such that, for any $i=1, \ldots, d$ and any $x \in \mathbb{T}^{N}$,

$$
\theta^{i}(x) \geq \theta_{0}>0
$$

Thus, we use

$$
\theta^{i}=g^{-1}\left(H^{i}\left(D u^{i}, x\right)+u^{i}+\sum_{j \neq i} \beta_{\epsilon}\left(u^{i}-u^{j}-\psi^{i j}\right)\right)
$$

in (1.5) to get

$$
\begin{aligned}
& -\theta^{i} H_{p_{k} p_{j}}^{i} u_{x_{j} x_{k}}^{i}-\theta^{i} H_{p_{k} x_{k}}^{i}-\frac{1}{g^{\prime}\left(\theta^{i}\right)}\left[H_{p_{k}}^{i} H_{p_{j}}^{i} u_{x_{j} x_{k}}^{i}+H_{x_{k}}^{i} H_{p_{k}}^{i}+H_{p_{k}}^{i} u_{x_{k}}^{i}\right. \\
& \left.+\sum_{j \neq i} \beta_{\epsilon}^{\prime}\left(u^{i}-u^{j}-\psi^{i j}\right)\left(u^{i}-u^{j}-\psi^{i j}\right)_{x_{k}} H_{p_{k}}^{i}\right]+\theta^{i}+\sum_{j \neq i} \beta_{\epsilon}^{\prime}\left(u^{i}-u^{j}-\psi^{i j}\right) \theta^{i} \\
& =\sum_{j \neq i} \beta_{\epsilon}^{\prime}\left(u^{j}-u^{i}-\psi^{j i}\right) \theta^{j}+1 .
\end{aligned}
$$

From estimates (5.1), (5.2), and (5.12), we have that the previous equation is a uniformly elliptic equation for each $i$. Therefore, from the elliptic regularity theory, we infer that

$$
\left\|u^{i}\right\|_{W^{2, p}\left(\mathbb{T}^{N}\right)}+\left\|\theta^{i}\right\|_{W^{1, p}\left(\mathbb{T}^{N}\right)} \leq C_{\epsilon}
$$

for any $1<p<\infty$. Repeated differentiation and a bootstrapping argument give (5.11). 


\section{Proof of Theorem 1.2}

In this section, we show the existence and uniqueness of a classical solution of (1.4)-(1.5). In the proof of existence, we use the continuation method. In the proof of uniqueness, we rely on a monotonicity argument. Here, we work under Assumptions 1-4 and either $5 \mathbf{L}$ or $5 \mathbf{P}-\frac{2}{N}$ together with Assumptions 6 and 7.

6.1. Existence. To prove the existence of a classical solution of (1.4)-(1.5) using the continuation method, we define

$$
H_{\lambda}^{i}(p, x):=\lambda H^{i}(p, x)+(1-\lambda) \frac{|p|^{2}}{2}
$$

for $0 \leq \lambda \leq 1,(p, x) \in \mathbb{R}^{N} \times \mathbb{T}^{N}$, and $i=1, \ldots, d$. We introduce the mean-field game

$$
\begin{gathered}
H_{\lambda}^{i}\left(D u_{\lambda}^{i}, x\right)+u_{\lambda}^{i}+\sum_{j \neq i} \beta_{\epsilon}\left(u_{\lambda}^{i}-u_{\lambda}^{j}-\psi^{i j}\right)=g\left(\theta_{\lambda}^{i}\right) \\
-\operatorname{div}\left(D_{p} H_{\lambda}^{i}\left(D u_{\lambda}^{i}, x\right) \theta_{\lambda}^{i}\right)+\theta_{\lambda}^{i}+\sum_{j \neq i} \beta_{\epsilon}^{\prime}\left(u_{\lambda}^{i}-u_{\lambda}^{j}-\psi^{i j}\right) \theta_{\lambda}^{i}-\beta_{\epsilon}^{\prime}\left(u_{\lambda}^{j}-u_{\lambda}^{i}-\psi^{j i}\right) \theta_{\lambda}^{j}=1
\end{gathered}
$$

for $x \in \mathbb{T}^{N}$ and $i=1, \ldots, d$.

Next, for $k \in \mathbb{N}$, we set $E^{k}:=\left(H^{k}\left(\mathbb{T}^{N}\right)\right)^{d}, E^{0}:=\left(L^{2}\left(\mathbb{T}^{N}\right)\right)^{d}$. If $k>\frac{N}{2}, E^{k}$ is an algebra. Moreover, $E^{k} \subset\left(C^{\gamma}\left(\mathbb{T}^{N}\right)\right)^{d}$ for any $0<\gamma<2-\frac{N}{k}$. Given $\theta_{0}>0$ and $k>\frac{N}{2}$, we set

$$
E_{\theta_{0}}^{k}:=\left\{\theta \in E^{k} \mid \theta^{i} \geq \theta_{0}, i=1, \ldots, d\right\} .
$$

Finally, for $k>\frac{N}{2}$, we define $F:[0,1] \times E^{k+2} \times E_{\theta_{0}}^{k+1} \rightarrow E^{k} \times E^{k+1}$ by

$$
\begin{aligned}
& F(\lambda, u, \theta):= \\
& \left(\begin{array}{c}
\operatorname{div}\left(D_{p} H_{\lambda}^{i}\left(D u_{\lambda}^{i}, x\right) \theta_{\lambda}^{i}\right)-\theta_{\lambda}^{i}-\sum_{j \neq i}\left(\beta_{\epsilon, \lambda}^{\prime}\left(u_{\lambda}^{i}-u_{\lambda}^{j}-\psi^{i j}\right) \theta_{\lambda}^{i}-\beta_{\epsilon, \lambda}^{\prime}\left(u_{\lambda}^{j}-u_{\lambda}^{i}-\psi^{j i}\right) \theta_{\lambda}^{j}\right)+1 \\
H_{\lambda}^{i}\left(D u_{\lambda}^{i}, x\right)+u_{\lambda}^{i}+\sum_{j \neq i} \beta_{\epsilon}\left(u_{\lambda}^{i}-u_{\lambda}^{j}-\psi^{i j}\right)-g\left(\theta_{\lambda}^{i}\right)
\end{array}\right) .
\end{aligned}
$$

Then, (6.1)-(6.2) is equivalent to

$$
F\left(\lambda, u_{\lambda}, \theta_{\lambda}\right)=0 .
$$

Let

$$
\Lambda:=\left\{\lambda \in[0,1] \mid(6.1)-(6.2) \text { has a classical solution }\left(u_{\lambda}, \theta_{\lambda}\right)\right\} .
$$

Next, we show that

$$
\Lambda=[0,1] .
$$

We divide the proof of this identity into the three following claims.

Claim 1: $0 \in \Lambda$. Indeed, for $\lambda=0$, we have the explicit solution:

$$
u_{0}^{i}=g(1), \quad \theta_{0}^{i}=1, \quad i=1, \ldots, d .
$$

Claim 2: $\Lambda$ is closed. To prove this claim, we show that, for any sequence, $\left(\lambda_{k}\right)_{k} \subset \Lambda$, such that $\lambda_{k} \rightarrow \lambda_{0}$ as $k \rightarrow+\infty$, we have that $\lambda_{0} \in \Lambda$. Accordingly, let $\left(u_{\lambda_{k}}, \theta_{\lambda_{k}}\right)$ be a classical solution of (6.1)-(6.2) for $\lambda=\lambda_{k}$. Recall that $H_{\lambda}$ satisfies Assumptions 1-4 and Assumption 6 uniformly in $0 \leq \lambda \leq 1$. Therefore, by (5.11), we can bound the derivatives of any order of $\left(u_{\lambda_{k}}, \theta_{\lambda_{k}}\right)$ by a constant that is independent of $k$. Consequently, we can extract a subsequence of smooth 
solutions converging to a limit function $(u, \theta)$ that solves (6.1)-(6.2) with $\lambda=\lambda_{0}$. Thus, $\lambda_{0} \in \Lambda$.

Claim 3: $\Lambda$ is open. To prove this last claim, we need to check that, for any $\lambda_{0} \in \Lambda$, there exists a neighborhood of $\lambda_{0}$ contained in $\Lambda$. To do so, we use the implicit function theorem. To simplify the notation, for $h=\beta, \beta^{\prime}, \beta^{\prime \prime}$, we set

$$
h_{\epsilon, \lambda_{0}}(i, j):=h_{\epsilon}\left(u_{\lambda_{0}}^{i}-u_{\lambda_{0}}^{j}-\psi^{i j}\right)
$$

For $\lambda_{0} \in \Lambda$, we consider the Fréchet derivative, $\mathcal{L}_{\lambda_{0}}: E^{k+2} \times E^{k+1} \rightarrow E^{k} \times E^{k+1}$, of $(u, \theta) \rightarrow F\left(\lambda_{0}, u, \theta\right)$ at $\left(u_{\lambda_{0}}, \theta_{\lambda_{0}}\right)$. We have

$$
\begin{aligned}
& \mathcal{L}_{\lambda_{0}}(v, f) \\
& =\left(\begin{array}{c}
\left(H_{\lambda_{0}, p_{k} p_{j}}^{i}\left(D u_{\lambda_{0}}^{i}, x\right) v_{x_{j}}^{i} \theta_{\lambda_{0}}^{i}+H_{\lambda_{0}, p_{k}}^{i}\left(D u_{\lambda_{0}}^{i}, x\right) f^{i}\right)_{x_{k}}-f^{i} \\
-\sum_{j \neq i}\left[\left(\beta_{\epsilon, \lambda_{0}}^{\prime \prime}(i, j) \theta_{\lambda_{0}}^{i}+\beta_{\epsilon, \lambda_{0}}^{\prime \prime}(j, i) \theta_{\lambda_{0}}^{j}\right)\left(v^{i}-v^{j}\right)+\beta_{\epsilon, \lambda_{0}}^{\prime}(i, j) f^{i}-\beta_{\epsilon, \lambda_{0}}^{\prime}(j, i) f^{j}\right] \\
D_{p} H_{\lambda_{0}}^{i}\left(D u_{\lambda_{0}}^{i}, x\right) \cdot D v^{i}+v^{i}+\sum_{j \neq i}\left(\beta_{\epsilon, \lambda_{0}}^{\prime}(i, j)\left(v^{i}-v^{j}\right)\right)-g^{\prime}\left(\theta_{\lambda_{0}}^{i}\right) f^{i}
\end{array}\right) .
\end{aligned}
$$

Because of the a priori bounds for smooth solutions (5.11) and either estimate (4.3), in the $\mathbf{L}$ case, or (4.12), in the $\mathbf{P}-\frac{2}{N}$ case, the operator, $\mathcal{L}_{\lambda_{0}}$, is well defined in $E^{k+2} \times E^{k+1}$ for any $k \geq 0$. Next, we prove that $\mathcal{L}_{\lambda_{0}}$ is an isomorphism from $E^{k+2} \times E^{k+1}$ to $E^{k} \times E^{k+1}$ for any $k \geq 0$.

Let $w=(v, f) \in E^{1} \times E^{0}$. Define the bilinear form, $B_{\lambda_{0}}\left[w_{1}, w_{2}\right]: E^{1} \times E^{0} \rightarrow \mathbb{R}$, by

$$
B_{\lambda_{0}}\left[w_{1}, w_{2}\right]:=\sum_{i=1}^{d} B_{\lambda_{0}}^{i}\left[w_{1}, w_{2}\right]
$$

where

$$
\begin{aligned}
& B_{\lambda_{0}}^{i}\left[w_{1}, w_{2}\right]:= \\
& \int_{\mathbb{T}^{N}}-H_{\lambda_{0}, p_{k} p_{j}}^{i}\left(D u_{\lambda_{0}}^{i}, x\right) v_{1, x_{j}}^{i} v_{2, x_{k}}^{i} \theta_{\lambda_{0}}^{i}-D_{p} H_{\lambda_{0}}^{i}\left(D u_{\lambda_{0}}^{i}, x\right) \cdot D v_{2}^{i} f_{1}^{i}-f_{1}^{i} v_{2}^{i} d x \\
& -\int_{\mathbb{T}^{N}}\left[\sum_{j \neq i}\left(\beta_{\epsilon, \lambda_{0}}^{\prime \prime}(i, j) \theta_{\lambda_{0}}^{i}+\beta_{\epsilon, \lambda_{0}}^{\prime \prime}(j, i) \theta_{\lambda_{0}}^{j}\right)\left(v_{1}^{i}-v_{1}^{j}\right)+\beta_{\epsilon, \lambda_{0}}^{\prime}(i, j) f_{1}^{i}-\beta_{\epsilon, \lambda_{0}}^{\prime}(j, i) f_{1}^{j}\right] v_{2}^{i} d x \\
& +\int_{\mathbb{T}^{N}}\left[D_{p} H_{\lambda_{0}}^{i}\left(D u_{\lambda_{0}}^{i}, x\right) \cdot D v_{1}^{i}+v_{1}^{i}+\sum_{j \neq i}\left(\beta_{\epsilon, \lambda_{0}}^{\prime}(i, j)\left(v_{1}^{i}-v_{1}^{j}\right)\right)-g^{\prime}\left(\theta_{\lambda_{0}}^{i}\right) f_{1}^{i}\right] f_{2}^{i} d x .
\end{aligned}
$$

If $w_{1} \in E^{k+2} \times E^{k+1}$ with $k \geq 0$, then

$$
B_{\lambda_{0}}\left[w_{1}, w_{2}\right]=\int_{\mathbb{T}^{N}} \mathcal{L}_{\lambda_{0}}\left(w_{1}\right) \cdot w_{2} d x
$$

The following lemma is a straightforward consequence of estimate (5.11) combined with either (4.3) or (4.12).

Lemma 6.1. Let $B$ be the bilinear form given by (6.4). Then, there exists $C>0$ such that

$$
\left|B_{\lambda_{0}}\left[w_{1}, w_{2}\right]\right| \leq C\left\|w_{1}\right\|_{E^{1} \times E^{0}}\left\|w_{2}\right\|_{E^{1} \times E^{0}}
$$

for any $w_{1}, w_{2} \in E^{1} \times E^{0}$. 
Thus, in view of Riesz's representation theorem for Hilbert spaces, there exists a continuous linear mapping, $A: E^{1} \times E^{0} \rightarrow E^{1} \times E^{0}$, such that

$$
B_{\lambda_{0}}\left[w_{1}, w_{2}\right]=\left(A w_{1}, w_{2}\right)_{E^{1} \times E^{0}} .
$$

Lemma 6.2. The operator, A, defined in (6.5) is injective.

Proof. Let $w=(v, f)$. Then, we have

$$
\begin{aligned}
& B_{\lambda_{0}}^{i}[w, w]=\int_{\mathbb{T}^{N}}-H_{\lambda_{0}, p_{k} p_{j}}^{i}\left(D u_{\lambda_{0}}^{i}, x\right) v_{x_{j}}^{i} v_{x_{k}}^{i} \theta_{\lambda_{0}}^{i} \\
& -\int_{\mathbb{T}^{N}} \sum_{j \neq i}\left(\beta_{\epsilon, \lambda_{0}}^{\prime \prime}(i, j) \theta_{\lambda_{0}}^{i}+\beta_{\epsilon, \lambda_{0}}^{\prime \prime}(j, i) \theta_{\lambda_{0}}^{j}\right)\left(v^{i}-v^{j}\right) v^{i}+g^{\prime}\left(\theta_{\lambda_{0}}^{i}\right)\left(f^{i}\right)^{2} d x .
\end{aligned}
$$

Summing the preceding expression on $i$, using the identity

$$
\begin{aligned}
& \sum_{i} \sum_{j \neq i}\left(\beta_{\epsilon, \lambda_{0}}^{\prime \prime}(i, j) \theta_{\lambda_{0}}^{i}+\beta_{\epsilon, \lambda_{0}}^{\prime \prime}(j, i) \theta_{\lambda_{0}}^{j}\right)\left(v^{i}-v^{j}\right) v^{i} \\
& =\sum_{i} \sum_{j \neq i} \beta_{\epsilon, \lambda_{0}}^{\prime \prime}(i, j) \theta_{\lambda_{0}}^{i}\left(v^{i}-v^{j}\right)^{2}
\end{aligned}
$$

the convexity property (2.4) from Assumption 4 and either (4.3), in the $\mathbf{L}$ case, or (4.12), in the $\mathbf{P}-\frac{2}{N}$ case, we get

$$
\begin{aligned}
B_{\lambda_{0}}[w, w] & =\sum_{i=1}^{d} B_{\lambda_{0}}^{i}[w, w] \\
& \leq-\sum_{i=1}^{d} \int_{\mathbb{T}^{N}} H_{\lambda_{0}, p_{k} p_{j}}^{i}\left(D u_{\lambda_{0}}^{i}, x\right) v_{x_{j}}^{i} v_{x_{k}}^{i} \theta_{\lambda_{0}}^{i}+g^{\prime}\left(\theta_{\lambda_{0}}^{i}\right)\left(f^{i}\right)^{2} d x \\
& -\sum_{i} \sum_{j \neq i} \int_{\mathbb{T}^{N}} \beta_{\epsilon, \lambda_{0}}^{\prime \prime}(i, j) \theta_{\lambda_{0}}^{i}\left(v^{i}-v^{j}\right)^{2} \\
& \leq-C_{\lambda_{0}} \int_{\mathbb{T}^{N}}\|D v\|^{2}+\|f\|^{2} d x \\
& -\theta_{0} \sum_{i} \sum_{j \neq i} \int_{\mathbb{T}^{N}} \beta_{\epsilon, \lambda_{0}}^{\prime \prime}(i, j)\left(v^{i}-v^{j}\right)^{2} d x .
\end{aligned}
$$

According to the previous inequality, if $A w=0$, we have $w=(\mu, 0)$ for some $\mu \in \mathbb{R}^{d}$. Next, by computing

$$
0=(A w,(0, \mu))=B[(\mu, 0),(0, \mu)]=\sum_{i=1}^{d} \int_{\mathbb{T}^{N}} v_{1}^{i} f_{2}^{i} d x=|\mu|^{2},
$$

we conclude that $\mu=0$.

Lemma 6.3. The operator, A, given by (6.5) is surjective.

Proof. First, we prove that the range of $A$ is closed in $E^{1} \times E^{0}$. For that, take a Cauchy sequence, $\left(z_{n}\right)_{n}$, in the range of $A$; that is, $z_{n}=A w_{n}$ for some sequence $\left(w_{n}\right)_{n}$ in $E^{1} \times E^{0}$. We claim that $\left(w_{n}\right)_{n}$ is a Cauchy sequence. Let $w_{n}=\left(v_{n}, f_{n}\right)$. 
Then, according to (6.6), we have

$$
\begin{aligned}
\left(z_{n}-z_{m}, w_{n}-w_{m}\right)_{E^{1} \times E^{0}}= & \left(A\left(w_{n}-w_{m}\right), w_{n}-w_{m}\right)_{E^{1} \times E^{0}} \\
& =B\left[w_{n}-w_{m}, w_{n}-w_{m}\right] \\
\leq & -C\left(\left\|D\left(v_{n}-v_{m}\right)\right\|_{E^{0}}^{2}+\left\|f_{n}-f_{m}\right\|_{E^{0}}^{2}\right) \\
& -\sum_{i} \sum_{j \neq i} \int_{\mathbb{T}^{N}} \beta_{\epsilon, \lambda_{0}}^{\prime \prime}(i, j)\left(\left(v_{n}^{i}-v_{m}^{i}\right)-\left(v_{n}^{j}-v_{m}^{j}\right)\right)^{2} d x
\end{aligned}
$$

Moreover, we have

$$
\begin{aligned}
& \left|\left(z_{n}-z_{m}, w_{n}-w_{m}\right)_{E^{1} \times E^{0}}\right| \leq\left\|z_{n}-z_{m}\right\|_{E^{1} \times E^{0}}\left\|w_{n}-w_{m}\right\|_{E^{1} \times E^{0}} \\
& \quad=\left\|z_{n}-z_{m}\right\|_{E^{1} \times E^{0}}\left(\left\|v_{n}-v_{m}\right\|_{E^{0}}+\left\|D v_{n}-D v_{m}\right\|_{E^{0}}+\left\|f_{n}-f_{m}\right\|_{E^{0}}\right) \\
& \quad \leq \delta\left(\left\|D v_{n}-D v_{m}\right\|_{E^{0}}^{2}+\left\|f_{n}-f_{m}\right\|_{E^{0}}^{2}\right)+C_{\delta}\left\|z_{n}-z_{m}\right\|_{E^{1} \times E^{0}}^{2} \\
& \quad+\left\|z_{n}-z_{m}\right\|_{E^{1} \times E^{0}}\left\|v_{n}-v_{m}\right\|_{E^{0}} .
\end{aligned}
$$

Let $\mu$ be a positive constant to be chosen later. By selecting a suitably small $\delta$ and combining the inequalities above, we get

$$
\begin{aligned}
& \left\|D v_{n}-D v_{m}\right\|_{E^{0}}^{2}+\left\|f_{n}-f_{m}\right\|_{E^{0}}^{2} \\
& +\sum_{i} \sum_{j \neq i} \int_{\mathbb{T}^{N} \cap\left\{u^{i}-u^{j}-\psi^{i j}>0\right\}} \beta_{\epsilon, \lambda_{0}}^{\prime \prime}(i, j)\left(\left(v_{n}^{i}-v_{m}^{i}\right)-\left(v_{n}^{j}-v_{m}^{j}\right)\right)^{2} d x \\
& \quad \leq C\left\|z_{n}-z_{m}\right\|_{E^{1} \times E^{0}}^{2}+\left\|z_{n}-z_{m}\right\|_{E^{1} \times E^{0}}\left\|v_{n}-v_{m}\right\|_{E^{0}} \\
& \quad \leq C_{\mu}\left\|z_{n}-z_{m}\right\|_{E^{1} \times E^{0}}^{2}+\mu\left\|v_{n}-v_{m}\right\|_{E^{0}}^{2} .
\end{aligned}
$$

Next, we have

$$
\begin{aligned}
& B\left[w_{n}-w_{m},\left(0, v_{n}-v_{m}\right)\right]= \\
& \qquad \int_{\mathbb{T}^{N}} \sum_{i=1}^{d}\left[D_{p} H_{\lambda_{0}}^{i}\left(D u_{\lambda_{0}}^{i}, x\right) \cdot\left(D\left(v_{n}^{i}-v_{m}^{i}\right)\right)\left(v_{n}^{i}-v_{m}^{i}\right)+\left(v_{n}^{i}-v_{m}^{i}\right)^{2}\right] d x \\
& \quad+\int_{\mathbb{T}^{N}} \sum_{i=1}^{d} \sum_{i \neq j} \beta_{\epsilon, \lambda_{0}}^{\prime}(i, j)\left[\left(v_{n}^{i}-v_{m}^{i}\right)-\left(v_{n}^{j}-v_{m}^{j}\right)\right]\left(v_{n}^{i}-v_{m}^{i}\right) d x \\
& \quad-\int_{\mathbb{T}^{N}} \sum_{i=1}^{d} g^{\prime}\left(\theta_{\lambda_{0}}^{i}\right)\left(f_{n}^{i}-f_{n}^{i}\right)\left(v_{n}^{i}-v_{m}^{i}\right) d x .
\end{aligned}
$$

Set

$$
M=\left[\sum_{i=1}^{d} \int_{\mathbb{T}^{N}}\left(\sum_{i \neq j} \beta_{\epsilon, \lambda_{0}}^{\prime}(i, j)\left[\left(v_{n}^{i}-v_{m}^{i}\right)-\left(v_{n}^{j}-v_{m}^{j}\right)\right]\right)^{2}\right]^{1 / 2} .
$$

Then, using (5.1), (5.2), Hölder's inequality, and Young's inequality, we get

$$
\begin{aligned}
& B\left[w_{n}-w_{m},\left(0, v_{n}-v_{m}\right)\right] \\
& \geq\left\|v_{n}-v_{m}\right\|_{E^{0}}^{2} \\
& -C\left(\left\|D v_{n}-D v_{m}\right\|_{E^{0}}^{2}+\left\|v_{n}-v_{m}\right\|_{E^{0}}\left\|f_{n}-f_{m}\right\|_{E^{0}}+M\left\|v_{n}-v_{m}\right\|_{E^{0}}\right) \\
& \geq C\left\|v_{n}-v_{m}\right\|_{E^{0}}^{2}-C\left(\left\|D v_{n}-D v_{m}\right\|_{E^{0}}^{2}+\left\|f_{n}-f_{m}\right\|_{E^{0}}^{2}+M^{2}\right) .
\end{aligned}
$$


On the other hand, (6.5) implies that

$$
B\left[w_{n}-w_{m},\left(0, v_{n}-v_{m}\right)\right] \leq C\left\|z_{n}-z_{m}\right\|_{E^{1} \times E^{0}}\left\|v_{n}-v_{m}\right\|_{E^{0}} .
$$

Therefore,

$$
\begin{aligned}
& C\left\|v_{n}-v_{m}\right\|_{E^{0}}^{2}-C\left(\left\|D v_{n}-D v_{m}\right\|_{E^{0}}^{2}+\left\|f_{n}-f_{m}\right\|_{E^{0}}^{2}+M^{2}\right) \\
& \leq C\left\|z_{n}-z_{m}\right\|_{E^{1} \times E^{0}}\left\|v_{n}-v_{m}\right\|_{E^{0}} \\
& \leq C\left\|z_{n}-z_{m}\right\|_{E^{1} \times E^{0}}^{2}+\mu_{2}\left\|v_{n}-v_{m}\right\|_{E^{0}}^{2}
\end{aligned}
$$

for some $\mu_{2}$ to be selected later. Next, taking into account that

$$
M^{2} \leq C \sum_{i} \sum_{j \neq i} \int_{\mathbb{T}^{N}} \beta_{\epsilon, \lambda_{0}}^{\prime \prime}(i, j)\left[\left(v_{n}^{i}-v_{m}^{i}\right)-\left(v_{n}^{j}-v_{m}^{j}\right)\right]^{2} d x
$$

and using (6.7) and (6.8), we obtain

$$
\left\|v_{n}-v_{m}\right\|_{E^{0}}^{2} \leq C\left\|z_{n}-z_{m}\right\|_{E^{1} \times E^{0}}^{2} .
$$

Consequently, $v_{n}$ is a Cauchy sequence in $L^{2}$. Finally, from (6.7) we infer that $w_{n}$ is a Cauchy sequence in $E^{1} \times E^{0}$ because of the bound

$$
\left\|w_{n}-w_{m}\right\|_{E^{1} \times E^{0}}^{2} \leq C\left\|z_{n}-z_{m}\right\|_{E^{1} \times E^{0}}^{2} .
$$

The last inequality and the continuity of $A$ imply that $R(A)$ is closed.

Finally, we prove that $R(A)=E^{1} \times E^{0}$. Suppose that $R(A) \neq E^{1} \times E^{0}$. Since $R(A)$ is closed, there exists $z \in R(A)^{\perp}$ with $z \neq 0$, such that $B_{\lambda_{0}}[z, z]=0$. The argument in the proof of Lemma 6.2 implies that $z=0$, which is a contradiction.

Lemma 6.4. The operator, $\mathcal{L}_{\lambda_{0}}: E^{k+2} \times E^{k+1} \rightarrow E^{k} \times E^{k+1}$, given by (6.3) is an isomorphism for all $k \in \mathbb{N}$.

Proof. By Lemma $6.2, \mathcal{L}_{\lambda_{0}}$ is injective. Therefore, it suffices to prove that $\mathcal{L}_{\lambda_{0}}$ is surjective. To do so, we fix $w_{0} \in E^{0} \times E^{1}$ with $w_{0}=\left(v_{0}, f_{0}\right)$. We claim that there exists a solution, $w_{1} \in E^{2} \times E^{1}$, to $\mathcal{L}_{\lambda_{0}} w_{1}=w_{0}$.

Consider the bounded linear functional, $w \rightarrow\left(w_{0}, w\right)_{\left(E^{0}\right)^{2}}$, in $E^{1} \times E^{0}$. According to the Riesz representation theorem, there exists $\widetilde{w} \in E^{1} \times E^{0}$, such that $\left(w_{0}, w\right)_{\left(E^{0}\right)^{2}}=(\widetilde{w}, w)_{E^{1} \times E^{0}}$ for any $w \in E^{1} \times E^{0}$. In light of Lemmas 6.2 and 6.3, the operator $A$ is invertible in $E^{1} \times E^{0}$. We define $w_{1}:=A^{-1} \widetilde{w}$. Then, for any $w \in E^{1} \times E^{0}$,

$$
\left(A w_{1}, w\right)_{E^{1} \times E^{0}}=\left(w_{0}, w\right)_{E^{0} \times E^{0}} ;
$$

that is, $w_{1}=(v, f)$ is a weak solution of

$$
\left\{\begin{array}{l}
\left(H_{\lambda_{0}, p_{k} p_{j}}^{i}\left(D u_{\lambda_{0}}^{i}, x\right) v_{x_{j}}^{i} \theta_{\lambda_{0}}^{i}+H_{\lambda_{0}, p_{k}}^{i}\left(D u_{\lambda_{0}}^{i}, x\right) f^{i}\right)_{x_{k}}-f^{i} \\
-\sum_{j \neq i}\left[\left(\beta_{\epsilon, \lambda_{0}}^{\prime \prime}(i, j) \theta_{\lambda_{0}}^{i}+\beta_{\epsilon, \lambda_{0}}^{\prime \prime}(j, i) \theta_{\lambda_{0}}^{j}\right)\left(v^{i}-v^{j}\right)+\beta_{\epsilon, \lambda_{0}}^{\prime}(i, j) f^{i}-\beta_{\epsilon, \lambda_{0}}^{\prime}(j, i) f^{j}\right]=v_{0}^{i} \\
D_{p} H_{\lambda_{0}}^{i}\left(D u_{\lambda_{0}}^{i}, x\right) \cdot D v^{i}+v^{i}+\sum_{j \neq i}\left(\beta_{\epsilon, \lambda_{0}}^{\prime}(i, j)\left(v^{i}-v^{j}\right)\right)-g^{\prime}\left(\theta_{\lambda_{0}}^{i}\right) f^{i}=f_{0}^{i}
\end{array}\right.
$$

for $x \in \mathbb{T}^{N}$ and $i=1, \ldots, d$. From the second equation in (6.9), we obtain

$$
f^{i}=\left(g^{\prime}\left(\theta_{\lambda_{0}}^{i}\right)\right)^{-1}\left(D_{p} H_{\lambda_{0}}^{i}\left(D u_{\lambda_{0}}^{i}, x\right) \cdot D v^{i}+v^{i}+\sum_{j \neq i}\left(\beta_{\epsilon, \lambda_{0}}^{\prime}(i, j)\left(v^{i}-v^{j}\right)\right)-f_{0}^{i}\right) .
$$

Using (6.10) in the first equation of (6.9), we see that $v^{i}$ is a weak solution to 


$$
\begin{aligned}
& {\left[H_{\lambda_{0}, p_{k} p_{j}}^{i}\left(D u_{\lambda_{0}}^{i}, x\right) v_{x_{j}}^{i} \theta_{\lambda_{0}}^{i}+H_{\lambda_{0}, p_{k}}^{i}\left(D u_{\lambda_{0}}^{i}, x\right) H_{\lambda_{0}, p_{j}}^{i}\left(D u_{\lambda_{0}}^{i}, x\right) v_{x_{j}}^{i}\left(g^{\prime}\left(\theta_{\lambda_{0}}^{i}\right)\right)^{-1}\right]_{x_{k}}} \\
& =-\left[\left(g^{\prime}\left(\theta_{\lambda_{0}}^{i}\right)\right)^{-1}\left(v^{i}+\sum_{j \neq i}\left(\beta_{\epsilon, \lambda_{0}}^{\prime}(i, j) v^{i}-\beta_{\epsilon, \lambda_{0}}^{\prime}(i, j) v^{j}\right)-f_{0}^{i}\right)\right]_{x_{k}}+f^{i} \\
& +\sum_{j \neq i}\left[\left(\beta_{\epsilon, \lambda_{0}}^{\prime \prime}(i, j) \theta_{\lambda_{0}}^{i}+\beta_{\epsilon, \lambda_{0}}^{\prime \prime}(j, i) \theta_{\lambda_{0}}^{j}\right)\left(v^{i}-v^{j}\right)+\beta_{\epsilon, \lambda_{0}}^{\prime}(i, j) f^{i}-\beta_{\epsilon, \lambda_{0}}^{\prime}(j, i) f^{j}\right]+v_{0}^{i} .
\end{aligned}
$$

For any $i=1, \ldots, d$, the right-hand side of the previous equation is in $L^{2}\left(\mathbb{T}^{N}\right)$. Thus, the elliptic regularity theory implies that $v \in E^{2}$. Consequently, (6.10) gives $f \in E^{1}$.

By induction, if $w_{0}=\left(v_{0}, f_{0}\right) \in E^{k} \times E^{k+1}$, then $w_{1}=(v, f) \in E^{k+2} \times E^{k+1}$. This concludes the proof of the lemma.

Claim 3 is now a straightforward consequence of Lemma 6.4 combined with the implicit function theorem in Banach spaces, see, for instance, [15].

Finally, we gather the previous results to prove the existence claim in Theorem 1.2 .

Proof of Theorem 1.2 - existence. Claims 1-3 imply that $\Lambda=[0,1]$. In particular $1 \in \Lambda$, i.e., there exists a $C^{\infty}$ solution of (1.4)-(1.5).

6.2. Uniqueness. Here, we complete the proof of Theorem 1.2 by proving the uniqueness of solutions for (1.4)-(1.5) using the monotonicity method.

Proof of Theorem 1.2 - uniqueness. Let $\left(u_{1}, \theta_{1}\right)$ and $\left(u_{2}, \theta_{2}\right)$ be classical solutions of (1.4)-(1.5). First, we take (1.4) with $(u, \theta)=\left(u_{1}, \theta_{1}\right)$ and $(u, \theta)=\left(u_{2}, \theta_{2}\right)$ and subtract the corresponding equations. Next, we multiply by $\theta_{1}^{i}-\theta_{2}^{i}$ and integrate. Accordingly, we obtain

$$
\begin{aligned}
& \int_{\mathbb{T}^{N}}\left[H^{i}\left(D u_{1}^{i}, x\right)-H^{i}\left(D u_{2}^{i}, x\right)\right]\left(\theta_{1}^{i}-\theta_{2}^{i}\right)+\left(u_{1}^{i}-u_{2}^{i}\right)\left(\theta_{1}^{i}-\theta_{2}^{i}\right) d x \\
& +\sum_{j \neq i} \int_{\mathbb{T}^{N}}\left(\beta_{\epsilon}\left(u_{1}^{i}-u_{1}^{j}-\psi^{i j}\right)-\beta_{\epsilon}\left(u_{2}^{i}-u_{2}^{j}-\psi^{i j}\right)\right)\left(\theta_{1}^{i}-\theta_{2}^{i}\right) d x \\
& =\int_{\mathbb{T}^{N}}\left(g\left(\theta_{1}^{i}\right)-g\left(\theta_{2}^{i}\right)\right)\left(\theta_{1}^{i}-\theta_{2}^{i}\right) d x .
\end{aligned}
$$

Now, we take (1.5) with $(u, \theta)=\left(u_{1}, \theta_{1}\right)$ and $(u, \theta)=\left(u_{2}, \theta_{2}\right)$. Next, we subtract the corresponding equations, multiply by $u_{1}^{i}-u_{2}^{i}$, and integrate by parts. Accordingly, we get

$$
\begin{aligned}
0 & =\int_{\mathbb{T}^{N}}\left(D_{p} H^{i}\left(D u_{1}^{i}, x\right) \theta_{1}^{i}-D_{p} H^{i}\left(D u_{2}^{i}, x\right) \theta_{2}^{i}\right) D\left(u_{1}^{i}-u_{2}^{i}\right)+\left(u_{1}^{i}-u_{2}^{i}\right)\left(\theta_{1}^{i}-\theta_{2}^{i}\right) d x \\
& +\sum_{j \neq i} \int_{\mathbb{T}^{N}}\left[\beta_{\epsilon}^{\prime}\left(u_{1}^{i}-u_{1}^{j}-\psi^{i j}\right) \theta_{1}^{i}-\beta_{\epsilon}^{\prime}\left(u_{1}^{j}-u_{1}^{i}-\psi^{j i}\right) \theta_{1}^{j}\right. \\
& \left.-\left(\beta_{\epsilon}^{\prime}\left(u_{2}^{i}-u_{2}^{j}-\psi^{i j}\right) \theta_{2}^{i}-\beta_{\epsilon}^{\prime}\left(u_{2}^{j}-u_{2}^{i}-\psi^{j i}\right) \theta_{2}^{j}\right)\right]\left(u_{1}^{i}-u_{2}^{i}\right) d x .
\end{aligned}
$$


Finally, we subtract the two previous identities, sum on $i$, and use the monotonicity of $g$ to conclude that

$$
\begin{aligned}
0 & \leq \sum_{i=1}^{d} \int_{\mathbb{T}^{N}}\left(g\left(\theta_{1}^{i}\right)-g\left(\theta_{2}^{i}\right)\right)\left(\theta_{1}^{i}-\theta_{2}^{i}\right) d x \\
& =\sum_{i=1}^{d} \int_{\mathbb{T}^{N}}\left[\left(H^{i}\left(D u_{1}^{i}, x\right)-H^{i}\left(D u_{2}^{i}, x\right)\right)\left(\theta_{1}^{i}-\theta_{2}^{i}\right)\right. \\
& \left.-\left(D_{p} H^{i}\left(D u_{1}^{i}, x\right) \theta_{1}-D_{p} H^{i}\left(D u_{2}^{i}, x\right) \theta_{2}\right) D\left(u_{1}^{i}-u_{2}^{i}\right)\right] d x \\
& +\sum_{i=1}^{d} \sum_{j \neq i} \int_{\mathbb{T}^{N}}\left(\beta_{\epsilon}\left(u_{1}^{i}-u_{1}^{j}-\psi^{i j}\right)-\beta_{\epsilon}\left(u_{2}^{i}-u_{2}^{j}-\psi^{i j}\right)\right)\left(\theta_{1}^{i}-\theta_{2}^{i}\right) d x \\
& -\sum_{i=1}^{d} \sum_{j \neq i} \int_{\mathbb{T}^{N}}\left[\beta_{\epsilon}^{\prime}\left(u_{1}^{i}-u_{1}^{j}-\psi^{i j}\right) \theta_{1}^{i}-\beta_{\epsilon}^{\prime}\left(u_{1}^{j}-u_{1}^{i}-\psi^{j i}\right) \theta_{1}^{j}\right. \\
- & \left.\left(\beta_{\epsilon}^{\prime}\left(u_{2}^{i}-u_{2}^{j}-\psi^{i j}\right) \theta_{2}^{i}-\beta_{\epsilon}^{\prime}\left(u_{2}^{j}-u_{2}^{i}-\psi^{j i}\right) \theta_{2}^{j}\right)\right]\left(u_{1}^{i}-u_{2}^{i}\right) d x .
\end{aligned}
$$

Now, from the convexity of $\beta_{\epsilon}$, we infer that

$$
\begin{aligned}
& \sum_{i=1}^{d} \sum_{j \neq i} \int_{\mathbb{T}^{N}}\left(\beta_{\epsilon}\left(u_{1}^{i}-u_{1}^{j}-\psi^{i j}\right)-\beta_{\epsilon}\left(u_{2}^{i}-u_{2}^{j}-\psi^{i j}\right)\right)\left(\theta_{1}^{i}-\theta_{2}^{i}\right) d x \\
& -\sum_{i=1}^{d} \sum_{j \neq i} \int_{\mathbb{T}^{N}}\left[\beta_{\epsilon}^{\prime}\left(u_{1}^{i}-u_{1}^{j}-\psi^{i j}\right) \theta_{1}^{i}-\beta_{\epsilon}^{\prime}\left(u_{1}^{j}-u_{1}^{i}-\psi^{j i}\right) \theta_{1}^{j}\right. \\
& \left.-\left(\beta_{\epsilon}^{\prime}\left(u_{2}^{i}-u_{2}^{j}-\psi^{i j}\right) \theta_{2}^{i}-\beta_{\epsilon}^{\prime}\left(u_{2}^{j}-u_{2}^{i}-\psi^{j i}\right) \theta_{2}^{j}\right)\right]\left(u_{1}^{i}-u_{2}^{i}\right) d x \\
& =\sum_{i=1}^{d} \sum_{j \neq i} \int_{\mathbb{T}^{N}}\left(\beta_{\epsilon}\left(u_{1}^{i}-u_{1}^{j}-\psi^{i j}\right)-\beta_{\epsilon}\left(u_{2}^{i}-u_{2}^{j}-\psi^{i j}\right)\right)\left(\theta_{1}^{i}-\theta_{2}^{i}\right) d x \\
& -\sum_{i=1}^{d} \sum_{j \neq i} \int_{\mathbb{T}^{N}}\left[\beta_{\epsilon}^{\prime}\left(u_{1}^{i}-u_{1}^{j}-\psi^{i j}\right) \theta_{1}^{i}-\beta_{\epsilon}^{\prime}\left(u_{2}^{i}-u_{2}^{j}-\psi^{i j}\right) \theta_{2}^{i}\right]\left(u_{1}^{i}-u_{2}^{i}-u_{1}^{j}+u_{2}^{j}\right) d x \\
& =-\sum_{i=1}^{d} \sum_{j \neq i} \int_{\mathbb{T}^{N}}\left[\beta_{\epsilon}\left(u_{1}^{i}-u_{1}^{j}-\psi^{i j}\right)-\beta_{\epsilon}\left(u_{2}^{i}-u_{2}^{j}-\psi^{i j}\right)\right. \\
& \left.-\beta_{\epsilon}^{\prime}\left(u_{2}^{i}-u_{2}^{j}-\psi^{i j}\right)\left(u_{1}^{i}-u_{1}^{j}-u_{2}^{i}+u_{2}^{j}\right)\right] \theta_{2}^{i} d x \\
& -\sum_{i=1}^{d} \sum_{j \neq i} \int_{\mathbb{T}^{N}}\left[\beta_{\epsilon}\left(u_{2}^{i}-u_{2}^{j}-\psi^{i j}\right)-\beta_{\epsilon}\left(u_{1}^{i}-u_{1}^{j}-\psi^{i j}\right)\right. \\
& \left.-\beta_{\epsilon}^{\prime}\left(u_{1}^{i}-u_{1}^{j}-\psi^{i j}\right)\left(u_{2}^{i}-u_{2}^{j}-u_{1}^{i}+u_{1}^{j}\right)\right] \theta_{1}^{i} d x \\
& \leq 0 .
\end{aligned}
$$


Moreover, using (2.4) of Assumption 4, we get

$$
\begin{aligned}
& \sum_{i=1}^{d} \int_{\mathbb{T}^{N}}\left[\left(H^{i}\left(D u_{1}^{i}, x\right)-H^{i}\left(D u_{2}^{i}, x\right)\right)\left(\theta_{1}^{i}-\theta_{2}^{i}\right)\right. \\
& \left.-\left(D_{p} H^{i}\left(D u_{1}^{i}, x\right) \theta_{1}-D_{p} H^{i}\left(D u_{2}^{i}, x\right) \theta_{2}\right) D\left(u_{1}^{i}-u_{2}^{i}\right)\right] d x \\
& =-\sum_{i=1}^{d} \int_{\mathbb{T}^{N}}\left[H^{i}\left(D u_{1}^{i}, x\right)-H^{i}\left(D u_{2}^{i}, x\right)-D_{p} H^{i}\left(D u_{2}^{i}, x\right) D\left(u_{1}^{i}-u_{2}^{i}\right)\right] \theta_{2}^{i} d x \\
& -\sum_{i=1}^{d} \int_{\mathbb{T}^{N}}\left[H^{i}\left(D u_{2}^{i}, x\right)-H^{i}\left(D u_{1}^{i}, x\right)-D_{p} H^{i}\left(D u_{1}^{i}, x\right) D\left(u_{2}^{i}-u_{1}^{i}\right)\right] \theta_{1}^{i} d x \\
& \leq-C \sum_{i=1}^{d} \int_{\mathbb{T}^{N}}\left|D\left(u_{1}^{i}-u_{2}^{i}\right)\right|^{2} d x .
\end{aligned}
$$

By combining the last two inequalities, we conclude that

$$
0 \leq-C \sum_{i=1}^{d} \int_{\mathbb{T}^{N}}\left|D\left(u_{1}^{i}-u_{2}^{i}\right)\right|^{2} d x .
$$

Thus, we infer that $u_{1}^{i}=u_{2}^{i}$ for any $i=1, \ldots, d$. Consequently,

$$
\begin{aligned}
\theta_{1}^{i} & =g^{-1}\left(H^{i}\left(D u_{1}^{i}, x\right)+u_{1}^{i}+\sum_{j \neq i} \beta_{\epsilon}\left(u_{1}^{i}-u_{2}^{j}-\psi^{i j}\right)\right) \\
& =g^{-1}\left(H^{i}\left(D u_{2}^{i}, x\right)+u_{2}^{i}+\sum_{j \neq i} \beta_{\epsilon}\left(u_{2}^{i}-u_{2}^{j}-\psi^{i j}\right)\right) \\
& =\theta_{2}^{i} .
\end{aligned}
$$

This concludes the proof of the uniqueness of the solution of (1.4)-(1.5).

\section{Proof of Theorem 1.1}

Now, we use Theorem 1.2 and a limiting procedure to prove Theorem 1.1.

Proof of Theorem 1.1. Let $\left(u_{\epsilon}, \theta_{\epsilon}\right)$ be the classical solution of (1.4)-(1.5), whose existence is guaranteed by Theorem 1.2. By estimates (4.5), (4.2), and (4.4) if Assumption 5 L holds, or by estimates (4.8), (4.14) and (4.13) if Assumptions $5 \mathbf{P}-\frac{2}{N}$ and 6 hold, there exist $\gamma \in(0,1), u \in\left(W^{2,2}\left(\mathbb{T}^{N}\right)\right)^{d} \cap\left(C^{\gamma}\left(\mathbb{T}^{N}\right)\right)^{d}$, and $\theta \in\left(W^{1,2}\left(\mathbb{T}^{N}\right)\right)^{d}$, such that, up to extracting a subsequence, as $\epsilon \rightarrow 0$,

$$
\begin{gathered}
u_{\epsilon} \rightarrow u \quad \text { in }\left(L^{\infty}\left(\mathbb{T}^{N}\right)\right)^{d}, \\
D u_{\epsilon} \rightarrow D u, \quad \theta_{\epsilon} \rightarrow \theta \quad \text { in }\left(L^{2}\left(\mathbb{T}^{N}\right)\right)^{d}, \\
D^{2} u_{\epsilon} \rightarrow D^{2} u, \quad \text { in }\left(L^{2}\left(\mathbb{T}^{N}\right)\right)^{d},
\end{gathered}
$$

and, for any $i=1, \ldots, d$ and $j \neq i$,

$$
u^{i}-u^{j}-\psi^{i j} \leq 0 \quad \text { in } \mathbb{T}^{N} .
$$


The uniform convergence of $u_{\epsilon}^{i}$ to $u^{i}$ implies that, if $x \in \mathbb{T}^{N}$ is such that $u^{i}(x)-$ $u^{j}(x)-\psi^{i j}(x)<0$, then, for a small enough $\epsilon$, we have $u_{\epsilon}^{i}(x)-u_{\epsilon}^{j}(x)-\psi^{i j}(x)<0$. Consequently,

$$
\beta_{\epsilon}\left(u_{\epsilon}^{i}(x)-u_{\epsilon}^{j}(x)-\psi^{i j}(x)\right)=\beta_{\epsilon}^{\prime}\left(u_{\epsilon}^{i}(x)-u_{\epsilon}^{j}(x)-\psi^{i j}(x)\right)=0 .
$$

We deduce that the limit, $(u, \theta)$, is a weak solution of

$$
\begin{gathered}
H^{i}\left(D u^{i}, x\right)+u^{i} \leq g\left(\theta^{i}\right) \quad \text { in } \mathbb{T}^{N}, \\
H^{i}\left(D u^{i}, x\right)+u^{i}=g\left(\theta^{i}\right) \quad \text { in } \cap_{j \neq i}\left\{u^{i}-u^{j}-\psi^{i j}<0\right\} .
\end{gathered}
$$

Next, for $j \neq i$, we introduce the measures

$$
\nu_{\epsilon}^{i j}=\beta_{\epsilon}^{\prime}\left(u_{\epsilon}^{i}(x)-u_{\epsilon}^{j}(x)-\psi^{i j}(x)\right) \theta^{j} .
$$

By (3.5), we have that $\int_{\mathbb{T}^{N}} \nu_{\epsilon}^{i j} d x \leq C$ for some constant, $C$, independent of $\epsilon$. Thus, there exist non-negative measures, $\nu^{i j}$, such that

$$
-\operatorname{div}\left(D_{p} H^{i}\left(D u^{i}, x\right) \theta^{i}\right)+\theta^{i}+\sum_{j \neq i}\left(\nu^{i j}-\nu^{j i}\right)=1 .
$$

Moreover, $\nu^{i j}$ is supported in the set $u^{i}-u^{j}-\psi^{i j}=0$.

\section{UNIQUENESS OF THE LIMIT}

In this last section, we discuss the uniqueness of the limit, $(u, \theta)$, in the proof of Theorem 1.1.

Proposition 8.1. Suppose that Assumptions 1-4 and 8 hold, and that either

- Assumption $5 \mathbf{L}$ or

- Assumptions $5 \mathbf{P}-\frac{2}{N}, 6$ and 7

hold. For $\epsilon>0$, let $\left(u_{\epsilon}, \theta_{\epsilon}\right)$ be the solution of (1.4)-(1.5). Then, the limit, $(u, \theta)$, as $\epsilon \rightarrow 0$ of the family $\left(u_{\epsilon}, \theta_{\epsilon}\right)$ exists; that is, $(u, \theta)$ is independent of the choice of subsequence.

Proof. For $k=0,1$, consider a sequence, $\epsilon_{n}^{k}$, converging to 0 . Let $\left(u_{k}, \theta_{k}\right)=$ $\lim _{n \rightarrow \infty}\left(u_{\epsilon_{n}^{k}}, \theta_{\epsilon_{n}^{k}}\right)$. By (1.1), we have

$$
H^{i}\left(D u_{k}^{i}, x\right)+u_{k}^{i}-g\left(\theta_{k}^{i}\right) \leq 0
$$

and

$$
u_{k}^{i}-u_{k}^{j}-\psi^{i j} \leq 0
$$

For $k=0,1$, let $\tilde{k}=1-k$. Taking into account the preceding inequalities and the uniform convexity of $H^{i}$, we have

$$
\begin{aligned}
0 \geq & H^{i}\left(D u_{k}^{i}, x\right)-g\left(\theta_{k}^{i}\right)+u_{k}^{i}+\sum_{j} \beta_{\epsilon_{n}^{\tilde{k}}}\left(u_{k}^{i}-u_{k}^{j}-\psi^{i j}\right) \\
\geq & H^{i}\left(D u_{\epsilon_{n}^{\tilde{k}}}^{i}, x\right)-g\left(\theta_{\epsilon_{\tilde{k}}^{\tilde{k}}}^{i}\right)+u_{\epsilon_{n}^{\tilde{k}}}^{i}+\sum_{j} \beta_{\epsilon_{n}^{\tilde{k}}}\left(u_{\epsilon_{\tilde{k}}^{i}}^{i}-u_{\epsilon_{n}^{\tilde{k}}}^{j}-\psi^{i j}\right) \\
& +u_{k}^{i}-u_{\epsilon_{n}^{\tilde{k}}}^{i}+D_{p} H^{i}\left(D u_{\epsilon_{n}^{\tilde{k}}}^{i}, x\right) \cdot D\left(u_{k}^{i}-u_{\epsilon_{n}^{i}}^{i}\right) \\
& +\sum_{j \neq i} \beta_{\epsilon_{n}^{\tilde{k}}}^{\prime}\left(u_{\epsilon_{n}^{i}}^{i}-u_{\epsilon_{n}^{\tilde{k}}}^{j}-\psi^{i j}\right)\left(\left(u_{k}^{i}-u_{\epsilon_{n}^{\tilde{k}}}^{i}\right)-\left(u_{k}^{j}-u_{\epsilon_{n}^{j}}^{j}\right)\right) \\
& +c \gamma\left|D\left(u_{k}^{i}-u_{\epsilon_{n}^{\tilde{k}}}^{i}\right)\right|^{2}+g\left(\theta_{\epsilon_{n}^{\tilde{k}}}^{i}\right)-g\left(\theta_{k}^{i}\right)
\end{aligned}
$$


for some $c>0$. Integrating with respect to $\theta_{\epsilon_{n}^{\tilde{k}}}^{i}$ and adding over $i$ and $k$ give

$$
\sum_{i, k} \int_{\mathbb{T}^{N}}\left[\gamma\left|D\left(u_{k}^{i}-u_{\epsilon_{n}^{\tilde{k}}}^{i}\right)\right|^{2} \theta_{\epsilon_{n}^{\tilde{k}}}^{i}+\left(g\left(\theta_{\epsilon_{n}^{\tilde{k}}}^{i}\right)-g\left(\theta_{k}^{i}\right)\right) \theta_{\epsilon_{n}^{\tilde{k}}}^{i}+u_{k}^{i}-u_{\epsilon_{n}^{\tilde{k}}}^{i}\right] d x \leq 0 .
$$

Because $\theta_{\epsilon_{n}^{\tilde{k}}}^{i}$ is bounded in $\left(W^{1,2}\left(\mathbb{T}^{N}\right)\right)^{d}$ and because $u_{\epsilon_{n}^{\tilde{k}}}$ is bounded in $\left(W^{2,2}\left(\mathbb{T}^{N}\right)\right)^{d}$, by extracting a further subsequence, if necessary, we have the following almost everywhere convergences:

$$
\left|D\left(u_{k}^{i}-u_{\epsilon_{n}^{\tilde{k}}}^{i}\right)\right|^{2} \theta_{\epsilon_{n}^{\tilde{k}}}^{i} \rightarrow\left|D\left(u_{k}^{i}-u_{\tilde{k}}^{i}\right)\right|^{2} \theta_{\tilde{k}}^{i}
$$

and

Furthermore,

$$
g\left(\theta_{\epsilon_{n}^{\tilde{n}}}^{i}\right) \theta_{\epsilon_{n}^{\tilde{k}}}^{i} \rightarrow g\left(\theta_{\tilde{k}}^{i}\right) \theta_{\tilde{k}}^{i}
$$

$$
\int_{\mathbb{T}^{N}} g\left(\theta_{k}^{i}\right) \theta_{\epsilon_{n}^{\tilde{k}}}^{i} d x \rightarrow \int_{\mathbb{T}^{N}} g\left(\theta_{k}^{i}\right) \theta_{\tilde{k}}^{i} d x
$$

Consequently, taking into account that

$$
\lim _{n \rightarrow \infty} \sum_{k} \int_{\mathbb{T}^{N}} u_{k}^{i}-u_{\epsilon_{n}^{\hat{k}}}^{i} d x=0
$$

and that $z \mapsto z g(z)$ is bounded by below, and using Fatou's Lemma, we obtain

$$
\sum_{i, k} \int_{\mathbb{T}^{N}} \gamma\left|D\left(u_{k}^{i}-u_{\tilde{k}}^{i}\right)\right|^{2} \theta_{\tilde{k}}^{i}+\left(g\left(\theta_{\tilde{k}}^{i}\right)-g\left(\theta_{k}^{i}\right)\right) \theta_{\tilde{k}}^{i} \leq 0
$$

Because $g$ is monotone increasing, the preceding inequality implies that $\theta_{1}=\theta_{2}$ and that $D u_{1}=D u_{2}$. Thus, using (1.1), we have $u_{1}=u_{2}$.

\section{REFERENCES}

[1] N. Al-Mulla, R. Ferreira, and D. Gomes. Two numerical approaches to stationary mean-field games. Preprint.

[2] S.A. Belbas. Optimal switching control of diffusion processes: The associated implicit variational problems. In Decision and Control including the Symposium on Adaptive Processes, 1981 20th IEEE Conference on, volume 20, pages 1380-1383, 1981.

[3] Fabio C., O. Ley, P. Loreti, and V. D. Nguyen. Large time behavior of weakly coupled systems of first-order Hamilton-Jacobi equations. NoDEA. Nonlinear Differential Equations and Applications, 19(6):719-749, 2012.

[4] F. Cagnetti, D. Gomes, H. Mitake, and H. V. Tran. A new method for large time behavior of degenerate viscous Hamilton-Jacobi equations with convex Hamiltonians. Ann. Inst. $H$. Poincaré Anal. Non Linéaire, 32(1):183-200, 2015.

[5] F. Cagnetti, D. Gomes, and H. V. Tran. Adjoint methods for obstacle problems and weakly coupled systems of PDE. ESAIM Control Optim. Calc. Var., 19(3):754-779, 2013.

[6] I. Capuzzo-Dolcetta and L. C. Evans. Optimal switching for ordinary differential equations. SIAM Journal of Control and Optimization, 22(1):143-161, 1984.

[7] I. Capuzzo-Dolcetta, M. Matzeu, and J.-L. Menaldi. On a system of first order quasivariational inequalities connected with optimal switching problem. Systems and Control Letters, 3:113-116, 1983.

[8] P. Cardaliaguet. Long time average of first order mean-field games and weak kam theory. Preprint, 2013.

[9] P. Cardaliaguet. Weak solutions for first order mean-field games with local coupling. Preprint, 2013.

[10] P. Cardaliaguet, P. Garber, A. Porretta, and D. Tonon. Second order mean field games with degenerate diffusion and local coupling. Preprint, 2014.

[11] P. Cardaliaguet and P. J. Graber. Mean field games systems of first order. ESAIM Control Optim. Calc. Var., 21(3):690-722, 2015. 
[12] M. Cirant. Multi-population Mean Field Games systems with Neumann boundary conditions. J. Math. Pures Appl. (9), 103(5):1294-1315, 2015.

[13] M. Cirant and G. Verzini. Bifurcation and segregation in quadratic two-populations mean field games systems. Preprint 2015.

[14] A. Davini and M. Zavidovique. Aubry sets for weakly coupled systems of Hamilton-Jacobi equations. SIAM J. Math. Anal., 46(5):3361-3389, 2014.

[15] J. Dieudonné. Foundations of modern analysis. Academic Press, New York-London, 1969. Enlarged and corrected printing, Pure and Applied Mathematics, Vol. 10-I.

[16] L. C. Evans. Some new PDE methods for weak KAM theory. Calc. Var. Partial Differential Equations, 17(2):159-177, 2003.

[17] L.C. Evans and A. Friedman. Optimal stochastic switching and the Dirichlet problem for the Bellman equation. Transactions of American Mathematical Society, 253:365-389, 1979.

[18] D. Farias, A. Figali, and D. Gomes. Weak kam theory for a weakly coupled system of hamiltonjacobi equations. Preprint, 2016.

[19] R. Ferreira and D. Gomes. Existence of weak solutions for stationary mean-field games through variational inequalities. Preprint.

[20] D. Gomes and H. Mitake. Existence for stationary mean-field games with congestion and quadratic Hamiltonians. NoDEA Nonlinear Differential Equations Appl., 22(6):1897-1910, 2015.

[21] D. Gomes, L. Nurbekyan, and M. Prazeres. Explicit solutions of one-dimensional first-order stationary mean-field games with a generic nonlinearity. Preprint, 2016.

[22] D. Gomes and S. Patrizi. Obstacle mean-field game problem. Interfaces Free Bound., 17(1):55-68, 2015.

[23] D. Gomes, S. Patrizi, and V. Voskanyan. On the existence of classical solutions for stationary extended mean field games. Nonlinear Anal., 99:49-79, 2014.

[24] D. Gomes and E. Pimentel. Time dependent mean-field games with logarithmic nonlinearities. To appear in SIAM Journal on Mathematical Analysis.

[25] D. Gomes and E. Pimentel. Local regularity for mean-field games in the whole space. To appear in Minimax Theory and its Applications, 2015.

[26] D. Gomes, E. Pimentel, and H. Sánchez-Morgado. Time dependent mean-field games in the superquadratic case. To appear in ESAIM: Control, Optimisation and Calculus of Variations.

[27] D. Gomes, E. Pimentel, and H. Sánchez-Morgado. Time-dependent mean-field games in the subquadratic case. Comm. Partial Differential Equations, 40(1):40-76, 2015.

[28] D. Gomes, E. Pimentel, and V. Voskanyan. Regularity theory for mean-field game systems. 2016.

[29] D. Gomes, G. E. Pires, and H. Sánchez-Morgado. A-priori estimates for stationary mean-field games. Netw. Heterog. Media, 7(2):303-314, 2012.

[30] D. Gomes and H. Sánchez Morgado. A stochastic Evans-Aronsson problem. Trans. Amer. Math. Soc., 366(2):903-929, 2014.

[31] D. Gomes and J. Saúde. Mean field games models - a brief survey. Dyn. Games Appl., 4(2):110-154, 2014.

[32] D. Gomes and A. Serra. Systems of weakly coupled Hamilton-Jacobi equations with implicit obstacles. Canadian Journal of Mathematics, 64:1289-1309, 2012.

[33] D. Gomes and V. Voskanyan. Short-time existence of solutions for mean-field games with congestion. To appear J. London Math. Soc.

[34] M. Huang, P. E. Caines, and R. P. Malhamé. Large-population cost-coupled LQG problems with nonuniform agents: individual-mass behavior and decentralized $\epsilon$-Nash equilibria. IEEE Trans. Automat. Control, 52(9):1560-1571, 2007.

[35] M. Huang, R. P. Malhamé, and P. E. Caines. Large population stochastic dynamic games: closed-loop McKean-Vlasov systems and the Nash certainty equivalence principle. Commun. Inf. Syst., 6(3):221-251, 2006.

[36] J.-M. Lasry and P.-L. Lions. Jeux à champ moyen. I. Le cas stationnaire. C. R. Math. Acad. Sci. Paris, 343(9):619-625, 2006.

[37] J.-M. Lasry and P.-L. Lions. Jeux à champ moyen. II. Horizon fini et contrôle optimal. C. $R$. Math. Acad. Sci. Paris, 343(10):679-684, 2006.

[38] J.-M. Lasry and P.-L. Lions. Mean field games. Jpn. J. Math., 2(1):229-260, 2007.

[39] J.-M. Lasry and P.-L. Lions. Mean field games. Cahiers de la Chaire Finance et Développement Durable, 2007. 
[40] H. Mitake, A. Siconolfi, H.V. Tran, and N. Yamada. A Lagrangian approach to weakly coupled Hamilton-Jacobi systems. ArXiv preprint, 2015. arxiv:1503.00521v1[math.AP].

[41] H. Mitake and H. V. Tran. Remarks on the large time behavior of viscosity solutions of quasi-monotone weakly coupled systems of Hamilton-Jacobi equations. Asymptotic Analysis, $77(1-2): 43-70,2012$.

[42] H. Mitake and H. V. Tran. A dynamical approach to the large-time behavior of solutions to weakly coupled systems of Hamilton-Jacobi equations. Journal de Mathématiques Pures et Appliquées. Neuvième Série, 101(1):76-93, 2014.

[43] H. Mitake and H. V. Tran. Homogenization of weakly coupled systems of Hamilton-Jacobi equations with fast switching rates. Archive for Rational Mechanics and Analysis, 211(3):733769, 2014.

[44] H. Mitake and Hung V. Tran. Weakly coupled systems of infinity laplace equations. Preprint, 2014.

[45] V. D. Nguyen. Some results on the large-time behavior of weakly coupled systems of first-order Hamilton-Jacobi equations. Journal of Evolution Equations, 14(2):299-331, 2014.

[46] A. Porretta. On the planning problem for the mean field games system. Dyn. Games Appl., 4(2):231-256, 2014.

[47] A. Porretta. Weak solutions to Fokker-Planck equations and mean field games. Arch. Ration. Mech. Anal., 216(1):1-62, 2015.

(D. A. Gomes) King Abdullah University of Science and Technology (KAUST), CEMSE Division , Thuwal 23955-6900. SAUdi Arabia.

E-mail address: diogo.gomes@kaust.edu.sa

(S. Patrizi) University of Texas at Austin, Austin, Texas, UsA

E-mail address: spatrizi@math.utexas.edu 